\title{
Neural dynamics of perceptual grouping: Textures, boundaries, and emergent segmentations
}

\author{
STEPHEN GROSSBERG and ENNIO MINGOLLA \\ Boston University, Boston, Massachusetts
}

\begin{abstract}
A real-time visual processing theory is used to analyze and explain a wide variety of perceptual grouping and segmentation phenomena, including the grouping of textured images, randomly defined images, and images built up from periodic scenic elements. The theory explains how "local" feature processing and "emergent" features work together to segment a scene, how segmentations may arise across image regions that do not contain any luminance differences, how segmentations may override local image properties in favor of global statistical factors, and why segmentations that powerfully influence object recognition may be barely visible or totally invisible. Network interactions within a Boundary Contour (BC) System, a Feature Contour (FC) System, and an Object Recognition (OR) System are used to explain these phenomena. The BC System is defined by a hierarchy of orientationally tuned interactions, which can be divided into two successive subsystems called the OC filter and the CC loop. The OC filter contains two successive stages of oriented receptive fields which are sensitive to different properties of image contrasts. The OC filter generates inputs to the CC loop, which contains successive stages of spatially short-range competitive interactions and spatially long-range cooperative interactions. Feedback between the competitive and cooperative stages synthesizes a global context-sensitive segmentation from among the many possible groupings of local featural elements. The properties of the BC System provide a unified explanation of several ostensibly different Gestalt rules. The BC System also suggests explanations and predictions concerning the architecture of the striate and prestriate visual cortices. The BC System embodies new ideas concerning the foundations of geometry, on-line statistical decision theory, and the resolution of uncertainty in quantum measurement systems. Computer simulations establish the formal competence of the $\mathrm{BC}$ System as a perceptual grouping system. The properties of the BC System are compared with probabilistic and artificial intelligence models of segmentation. The total network suggests a new approach to the design of computer vision systems, and promises to provide a universal set of rules for perceptual grouping of scenic edges, textures, and smoothly shaded regions.
\end{abstract}

\section{Introduction: Toward a Universal Set of Rules for Perceptual Grouping}

The visual system segments optical input into regions that are separated by perceived contours or boundaries. This rapid, seemingly automatic, early step in visual processing is difficult to characterize, largely because many perceived contours have no obvious correlates in the optical input. A contour in a pattern of luminances is generally defined as a spatial discontinuity in luminance. Although usually sufficient, however, such discontinuities are by no means necessary for sustaining perceived contours. Regions separated by visual contours also oc-

S. Grossberg was supported in part by the Air Force Office of Scientific Research (AFOSR 85-0149) and the Army Research Office (DAAG29-85-K-0095). E. Mingolla was supported in part by the Air Force Office of Scientific Research (AFOSR 85-0149). We wish to thank Cynthia Suchta for her valuable assistance in the preparation of the manuscript and illustrations.

The authors' mailing address is: Center for Adaptive Systems, Department of Mathematics, Boston University, Boston, MA 02215. cur in the presence of: statistical differences in textural qualities such as orientation, shape, density, or color (Beck, 1966a, 1966b, 1972, 1982, 1983; Beck, Prazdny, \& Rosenfeld, 1983), binocular matching of elements of differing disparities (Julesz, 1960), accretion and deletion of texture elements in moving displays (Kaplan, 1969), and classical "subjective contours" (Kanizsa, 1955). The extent to which the types of perceived contours just named involve the same visual processes as those triggered by luminance contours is not obvious, although the former are certainly as perceptually real and generally as vivid as the latter.

Perceptual contours arising at boundaries of regions with differing statistical distributions of featural qualities have been studied in great detail (Beck, 1966a, 1966b, 1972, 1982, 1983; Beck et al., 1983; Caelli, 1982, 1983; Caelli \& Julesz, 1979). Two findings of this research are especially salient. First, the visual system's segmentation of the scenic input occurs rapidly throughout all regions of that input, in a manner often described as "preattentive." That is, subjects generally describe boundaries in 
a consistent manner when exposure times are short (under $200 \mathrm{msec}$ ) and without prior knowledge of the regions in a display at which boundaries are likely to occur. Thus, any theoretical account of boundary extraction for such displays must exlain how early "data driven" processes rapidly converge on boundaries wherever they occur.

The second finding of the experimental work on textures complicates the implications of the first, however: the textural segmentation process is exquisitely contextsensitive. That is, a given texture element at a given location can be part of a variety of larger groupings, depending on what surrounds it. Indeed, the precise determination even of what acts as an element at a given location can depend on patterns at nearby locations.

One of the greatest sources of difficulty in understanding visual perception and in designing fast object recognition systems is such context sensitivity of perceptual units. Since the work of the Gestaltists (Wertheimer, 1923), it has been widely recognized that local features of a scene, such as edge positions, disparities, lengths, orientations, and contrasts, are perceptually ambiguous, but that combinations of these features can be quickly grouped by a perceiver to generate a clear separation between figures and between figure and ground. Indeed, a figure within a textured scene often seems to "pop out" from the ground (Neisser, 1967). The "emergent" features by which an observer perceptually groups the "local" features within a scene are sensitive to the global structuring of textural elements within the scene.

The fact that these emergent perceptual units, rather than local features, are used to group a scene carries with it the possibility of scientific chaos. If every scene can define its own context-sensitive units, then perhaps object perception can only be described in terms of an unwieldy taxonomy of scenes and their unique perceptual units. One of the great accomplishments of the Gestaltists was to suggest a short list of rules for perceptual grouping that helped to organize many interesting examples. As is often the case in pioneering work, the rules were neither always obeyed nor exhaustive. No justification for the rules was given other than their evident plausibility. More seriously for practical applications, no effective computational algorithms were given to instantiate the rules.

Many workers since the Gestaltists have made important progress in advancing our understanding of perceptual grouping processes. For example, Dev (1975), Julesz (1971), and Sperling (1970) introduced algorithms for using disparity cues to coherently separate figure from ground in random-dot stereograms. Later workers, such as Marr and Poggio (1976), have studied similar algorithms. Caelli $(1982,1983)$ has emphasized the importance of the conjoint action of orientation and spatial frequency tuning in the filtering operations that preprocess textured images. Caelli and Dodwell (1982), Dodwell (1983), and Hoffman (1970) have recommended the use of Lie group vector fields as a tool for grouping together orientational cues across perceptual space. Caelli and Julesz (1979) have presented evidence that "first order statistics of textons" are used to group textural elements. The term "textons" designates the features that are to be statistically grouped. This view supports a large body of work by Beck and his colleagues (Beck, 1966a, 1966b, 1972, 1982, 1983; Beck et al., 1983), who have introduced a remarkable collection of ingenious textural displays which they have used to determine some of the factors that control textural grouping properties.

The collective effect of these and other contributions has been to provide a sophisticated experimental literature about textural grouping which has identified the main properties that need to be considered. What has not been achieved is a deep analysis of the design principles and mechanisms that lie behind the properties of perceptual grouping. Expressed in another way, what is missing is the raison d'etre for textural grouping and a computational framework that dynamically explains how textural elements are grouped, in real time, into easily separated figures and ground.

One manifestation of this gap in contemporary understanding can be found in the image-processing models that have been developed by workers in artificial intelligence. In this approach, curves are analyzed using models different from those that are used to analyze textures, and textures are analyzed using models different from the ones used to analyze surfaces (Horn, 1977; Marr \& Hildreth, 1980). All of these models are built up using geometrical ideas-such as surface normal, curvature, and Laplacian-that were used to study visual perception during the 19th century (Ratliff, 1965). These geometrical ideas were originally developed to analyze local properties of physical processes. By contrast, the visual system's context-sensitive mechanisms routinely synthesize figural percepts that are not reducible to local luminance differences within a scenic image. Such emergent properties are not just the effect of local geometrical transformations.

Our recent work suggests that 19 th century geometrical ideas are fundamentally inadequate to characterize the designs that make biological visual systems so efficient (Carpenter \& Grossberg, 1981, 1983; Cohen \& Grossberg, 1984a, 1984b; Grossberg, 1983a, 1983b, 1984a, 1985; Grossberg \& Mingolla, 1985a, 1985b). This claim arises from the discovery of new mechanisms that are not designed to compute local geometrical properties of a scenic image. These mechanisms are defined by parallel and hierarchical interactions within very large networks of interacting neurons. The visual properties that these equations compute emerge from network interactions, rather than from local transformations.

A surprising consequence of our analysis is that the same mechanisms that are needed to achieve a biologically relevant understanding of how scenic edges are internally represented also respond intelligently to textured images, smoothly shaded images, and combinations thereof. These new designs thus promise to provide a 
universal set of rules for the preattentive perceptual grouping processes that feed into depthful form percept and object recognition processes.

The complete development of these designs will require a major scientific effort. The present article takes two steps in that direction. The first goal of the article is to indicate how these new designs render transparent properties of perceptual grouping which previously were effectively manipulated by a small number of scientists, notably Jacob Beck. A primary goal of this article is thus to provide a dynamic explanation of recent textural displays from the Beck school. Beck and his colleageus have gone far in determining which aspects of textures tend to group and under what conditions. Our work sheds light on how such segmentation may be implemented by the visual system. The results of Glass and Switkes (1976) on grouping of statistically defined percepts and of Gergory and Heard (1979) on border locking during the café wall illusion will also be analyzed using the same ideas. The second goal of the article is to report computer simulations that illustrate the theory's formal competence for generating perceptual groupings that strikingly resemble human grouping properties.

Our theory first introduced the distinction between the Boundary Contour System (BC System) and the Feature Contour System (FC System) to deal with paradoxical data concerning brightness, color, and form perception. These two systems extract two different types of contoursensitive information-called BC signals and FC signals-at an early processing stage. The $\mathrm{BC}$ signals are transformed through successive processing stages within the BC System into coherent boundary structures. These boundary structures give rise to topographically organized output signals to the FC System (Figure 1). FC signals are sensitive to luminance and hue differences within a scenic image. These signals activate the same processing stage within the FC System that receives boundary signals from the BC System. The FC signals here initiate the filling-in processes whereby brightnesses and colors spread until they either hit their first boundary contour or are attenuated by their spatial spread.

Although earlier work examined the role of the BC System in the synthesis of individual contours, whether "real" or "illusory,"' its rules also account for much of the segmentation of textured scenes into grouped regions separated by perceived contours. Accordingly, Sections 2-9 of this paper review the main points of the theory with respect to their implications for perceptual grouping. Sections 10-15 and 17-19 then examine in detail the major issues in grouping research to date and describe our solutions qualitatively. Section 16 presents computer simulations showing how our model synthesizes contextsensitive perceptual groupings. The model is described in more mechanistic detail in Section 20. Mathematical equations of the model are contained in the Appendix.

\section{The Role of Illusory Contours}

One of the main themes in our discussion is the role of illusory contours in perceptual grouping processes. Our

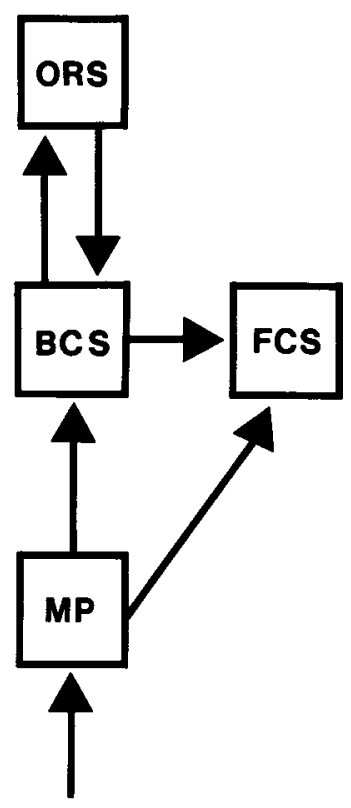

Figure 1. A macrocircuit of processing stages: Monocular preprocessed signals (MP) are sent independently to both the Boundary Contour System (BCS) and the Feature Contour System (FCS). The BCS preattentively generates coherent boundary structures from these MP signals. These structures send outputs to both the FCS and the Object Recognition System (ORS). The ORS, in turn, rapidly sends top-down learned template signals to the BCS. These template signals can modify the preattentively completed boundary structures using learned information. The BCS passes these modifications along to the FCS. The signals from the BCS organize the FCS into perceptual regions wherein filling-in of visible brightnesses and colors can occur. This filling-in process is activated by signals from the MP stage.

results make precise the sense in which percepts of "illusory contours" - or contour percepts that do not correspond to one-dimensional luminance differences in a scenic image-and percepts of "real contours" are both synthesized by the same mechanisms. This discussion clarifies why, despite the visual system's manifestly adaptive design, illusory contours are so abundant in visual percepts. We also suggest how illusory contours that are at best marginally visible can have powerful effects on perceptual grouping and object recognition processes.

Some of the new designs of our theory can be motivated by contrasting the noisy visual signals that reach the retina with the coherence of conscious visual percepts. In humans, for example, light passes through a thicket of retinal veins before it reaches retinal photoreceptors. The percepts of human observers are fortunately not distorted by their retinal veins during normal vision. This is due, in part, to the action of mechanisms that attenuate the perception of images that are stabilized with respect to the retina as the eye jiggles in its orbit with respect to the outside world. Suppressing the percept of the stabilized veins does not, in itself, complete the percept of retinal images that are occluded and segmented by the veins. Boundaries need to be completed and colors and brightnesses filled in to compensate for the image degradation 
that is caused by the retinal veins. A similar discussion follows from a consideration of why human observers do not typically notice their blind spots (Kawabata, 1984).

Observers are not able to distinguish which parts of such a completed percept are derived directly from retinal signals and which parts are due to boundary completion and featural filling-in. The completed and filled-in percepts are called, in the usual jargon, "illusory" figures. These examples suggest that both "real" and "illusory" figures are generated by the same perceptual mechanisms, and suggest why "illusory" figures are so important in perceptual grouping processes. Once this is understood, the need for a perceptual theory that treats "real" and "illusory" percepts on an equal footing also becomes apparent.

A central issue in such a theory concerns whether boundary completion and featural filling-in are the same or distinct processs. One of our theory's primary contributions is to show, by characterizing the different processing rules that they obey, that these processes are different.

At our present stage of understanding, many perceptual phenomena can be used to make this point. We find the following three phenomena to be particularly useful: the Land (1977) color and brightness experiments, the Yarbus (1967) stabilized-image experiments, and the reverse-contrast Kanizsa square (Grossberg \& Mingolla, 1985a).

\section{Discounting the Illuminant: Color Edges and Featural Filling-In}

The visual world is typically viewed under inhomogeneous lighting conditions. The scenic luminances that reach the retina thus confound fluctuating lighting conditions with invariant object colors and lightnesses. Helmholtz (1890/1962) aleady knew that the brain somehow "discounts the illuminant" to generate color and lightness percepts that are more veridical than those in the retinal image. Land (1977) has clarified this process in a series of striking experiments wherein color percepts within a picture constructed from overlapping patches of colored paper are determined under a variety of lighting conditions. These experiments show that color signals corresponding to the interior of each patch are suppressed. The chromatic contrasts across the edges between adjacent patches are used to generate the final percept. It is easy to see how such a scheme "discounts the illuminant." Large differences in illumination can exist within any patch. On the other hand, differences in illumination are small across an edge on such a planar display. Hence, the relative chromatic contrasts across edges, assumed to be registered by black-white, red-green, and blue-yellow double opponent systems, are good estimates of the object reflectances near the edge.

Just as suppressing the percept of stabilized veins is insufficient to generate an adequate percept, so too is discounting the illuminant within each color patch. Without further processing, we could at best perceive a world of colored edges. Featural filling-in is needed to recover estimates of brightness and color within the interior of each patch. Thus, extraction of color edges and featural fillingin are both necessary for the perception of a color field or a continuously shaded surface.

\section{Featural Filling-In Over Stabilized Scenic Edges}

Many images can be used to firmly establish that a featural filling-in process exists. The recent thesis of Todorović (1983) provides a nice set of examples that one can construct with modest computer graphics equipment. Vivid classical examples of featural filling-in were discovered by artificially stabilizing certain image contours of a scene (Krauskopf, 1963; Yarbus, 1967). Consider, for example, the image schematized in Figure 2. After the edges of the large circle and the vertical line are stabilized on the retina, the red color (dots) outside the large circle fills in the black and white hemidisks, except within the small red circles whose edges are not stabilized (Yarbus, 1967). The red inside the left circle looks brighter and the red inside the right circle looks darker than the uniform red that envelopes the remainder of the percept.

When the Land (1977) and Yarbus (1967) experiments are considered side by side, one can recognize that the brain extracts two different types of contour information from scenic images. Feature contours, including "color edges, " give rise to the signals that generate visible brightness and color percepts at a later processing stage. Feature contours encode this information as a contour-

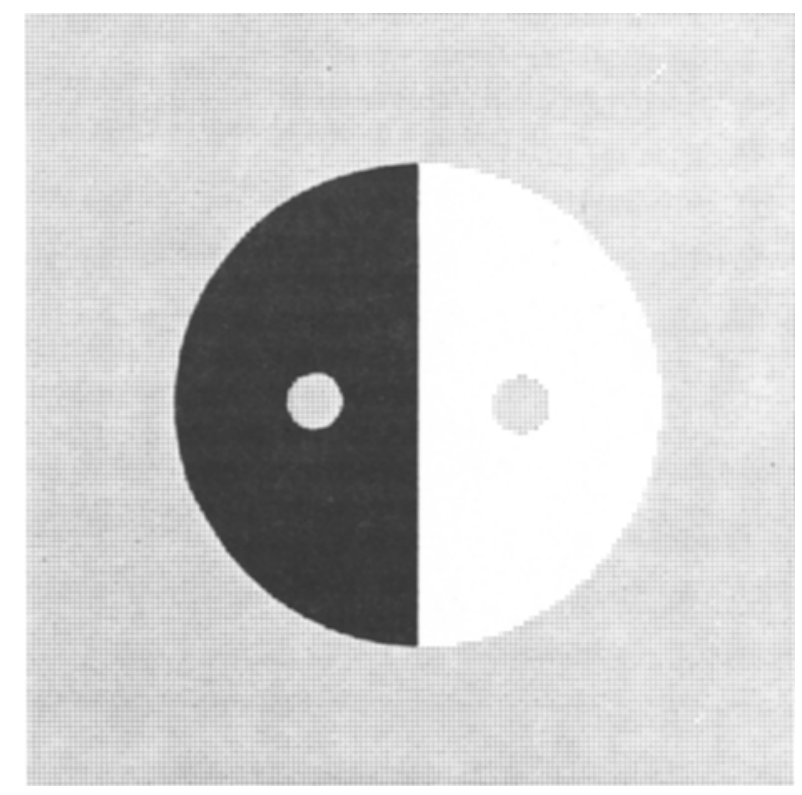

Figure 2. A classical example of featural filling-in: When the edges of the large circle and the vertical line are stabilized on the retina, the red color (dots) outside the large circle envelopes the black and white hemidisks except within the small red circles whose edges are not stabilized (Yarbus, 1967). The red inside the left circle looks brighter and the red inside the right circle looks darker than the enveloping red. 
sensitive process in order to discount the illuminant. Boundary contours are extracted in order to define the perceptual boundaries, groupings, or forms within which featural estimates derived from the feature contours can fill in at a later processing stage. In the Yarbus (1967) experiments, once a stabilized scenic edge can no longer generate a boundary contour, featural signals can flow across the locations corresponding to the stabilized scenic edge until they reach the next boundary contour. The phenomenon of neon color spreading also illustrates the dissociation of boundary-contour and feature-contour processing (Ejima, Redies, Takahashi, \& Akita, 1984; Redies \& Spillmann, 1981; Redies, Spillmann, \& Kunz, 1984; van Tuijl, 1975; van Tuijl \& de Weert, 1979; van Tuijl \& Leeuwenberg, 1979). An explanation of neon color spreading is suggested in Grossberg (1984a) and Grossberg and Mingolla (1985a).

\section{Different Rules for Boundary Contours and Feature Contours}

Some of the rules that distinguish the BC System from the FC System can be inferred from the percept generated by the reverse-contrast Kanizsa square image in Figure 3 (Cohen \& Grossberg, 1984a; Grossberg \& Mingolla, 1985a). Prazdny $(1983,1985)$ and Shapley and Gordon (1985) have also used reverse-contrast images in their discussions of form perception. Consider the vertical boundaries in the perceived Kanizsa square. In this percept, a vertical boundary connects a pair of vertical scenic edges with opposite direction of contrast. In other words, the black Pac-Man figure causes a dark-light vertical edge with respect to the gray background. The white Pac-Man figure causes a light-dark vertical edge with respect to the gray background. The process of boundary completion whereby a boundary contour is synthesized between these inducing stimuli is thus indifferent to direction of contrast. The boundary completion process is, however, sensitive to the orientation and amount of contrast of the inducing stimuli.

The feature contours extracted from a scene are, by contrast, exquisitely sensitive to direction of contrast. Were this not the case, we could never tell the difference between a dark-light and a light-dark percept. We would be blind.

Another difference between BC and FC rules can be inferred from Figures 2 and 3. In Figure 3, a boundary forms inward in an oriented way between a pair of inducing scenic edges. In Figure 2, featural filling-in is due to an outward and unoriented spreading of featural quality from individual FC signals that continues until the spreading signals either hit a boundary contour or are attenuated by their own spatial spread (Figure 4). The remainder of the article develops these and deeper properties of the BC System to explain segmentation data. Certain crucial points may profitably be emphasized now.

Boundaries may emerge corresponding to image regions in which no contrast differences whatsoever exist. The BC System is sensitive to statistical differences in the dis-

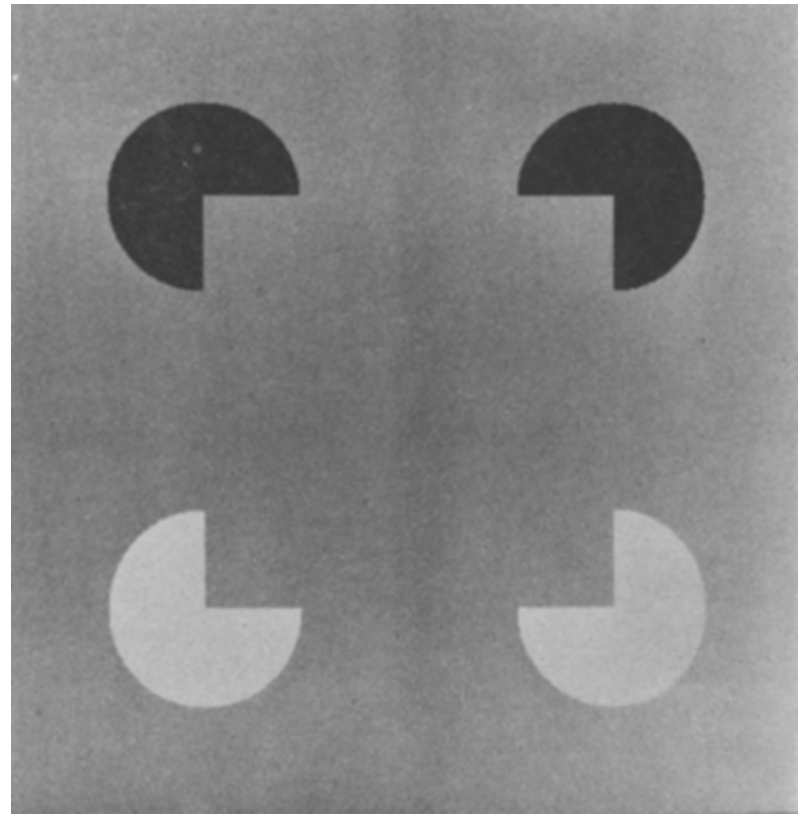

Figure 3. A reverse-contrast Kanisza square: An illusory square is induced by two black and two white Pac-Man figures on a gray background. Illusory contours can thus join edges with opposite directions of contrast. (This effect may be weakened by the photographic reproduction process.)

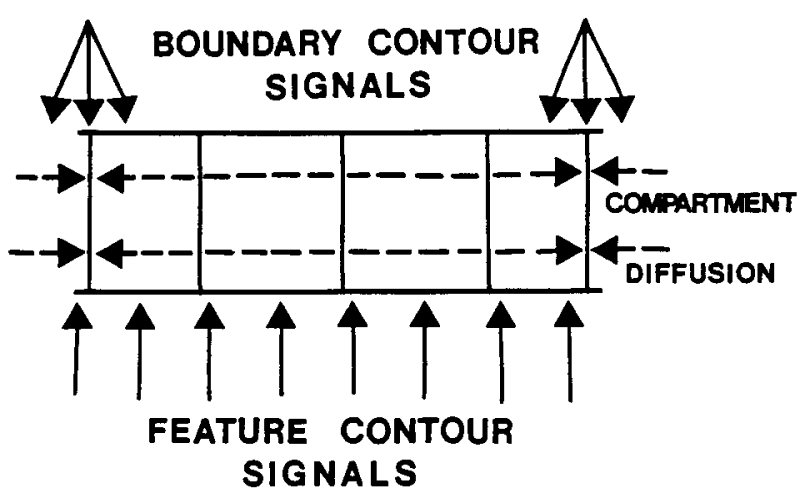

Figure 4. A monocular brightness and color stage domain within the FC System: Monocular feature contour signals activate cell compartments which permit rapid lateral diffusion of activity, or potential, across their compartment boundaries, except at those compartment boundaries which receive boundary contour signals from the BC System. Consequently, the FC signals are smoothed except at boundaries that are completed within the BC System stage.

tribution of scenic elements, not merely to individual image contrasts. In particular, the oriented receptive fields, or masks, that initiate boundary processing are not edge detectors; rather, they are local contrast detectors which can respond to statistical differences in the spatial distribution of image contrasts, including but not restricted to edges. These receptive fields are organized into multiple subsystems, such that the oriented receptive fields within 
each subsystem are sensitive to oriented contrasts over spatial domains of different sizes. These subsystems can therefore respond differently to spatial frequency information within the scenic image. Since all these oriented receptive fields are also sensitive to amount of contrast, the BC System registers statistical differences in luminance, orientation, and spatial frequency even at its earliest stages of processing.

Later stages of BC System processing are also sensitive to these factors, but in a different way. Their inputs from earlier stages are already sensitive to these factors. They then actively transform these inputs, using competitive-cooperative feedback interactions. The BC System may hereby process statistical differences in luminance, orientation, and spatial frequency within a scenic image in multiple ways.

We wish also to dispel misconceptions that a comparison between the names "Boundary Contour System" and "Feature Contour System"' may engender. As indicated above, the BC System does generate perceptual boundaries, but neither the data nor our theory permit the conclusion that these boundaries must coincide with the edges in scenic images. The FC System does lead to visible percepts, such as organized brightness and color differences, and such percepts contain the elements that are often called features.

On the other hand, both the BC System and the FC System contain "feature detectors" which are sensitive to luminance or hue differences within scenic images. Although both systems contain "feature detectors," these detectors are used within the BC System to generate boundaries, not visible "features." In fact, within the BC System, all boundaries are perceptually invisible.

Boundary contours do, however, contribute to visible percepts, but only indirectly. All visible percepts arise within the FC System. Completed boundary contours help to generate visible percepts within the FC System by defining the perceptual regions within which activations due to feature contour signals can fill in.

Our names for these two systems emphasize that conventional usage of the terms "boundary" and "feature" needs modification to explain data about form and color perception. Our usage of these important terms captures the spirit of their conventional meaning, but also refines this meaning, to be consistent within a mechanistic analysis of the interactions leading to form and color percepts.

\section{Boundary-Feature Tradeoff: Every Line End Is Illusory}

The rules obeyed by the BC System can be fully understood only by considering how they interact with the rules of the FC System. Each contour system is designed to offset insufficiencies of the other. The most paradoxical properties of the BC System can be traced to its role in defining the perceptual domains that restrict featural filling-in. These also turn out to be the properties that are most important in the regulation of perceptual grouping.
The inability of previous perceptual theories to provide a transparent analysis of perceptual grouping can be traced to the fact that they did not clearly distinguish boundary contours from feature contours; hence they could not adequately understand the rules whereby boundary contours generate perceptual groupings to define perceptual domains adequate to contain featural filling-in.

When one frontally assaults the problem of designing boundary contours to contain featural filling-in, one is led to many remarkable conclusions. One conclusion is that the end of every line is an "illusory" contour. We now summarize what we mean by this assertion.

An early stage of boundary-contour processing needs to determine the orientations in which scenic edges are pointing. This is accomplished by elongated receptive fields, or orientationally tuned input masks (Hubel \& Wiesel, 1977). Elongated receptive fields are, however, insensitive to orientation at the ends of thin lines and at object corners (Grossberg \& Mingolla, 1985a). This breakdown is illustrated by the computer simulation summarized in Figure 5a, which depicts the reaction of a lattice of orientationally tuned cells to a thin vertical line. Figure 5a shows that in order to achieve some measure of orientational certainty along scenic edges, the cells sacrifice their ability to determine either position or orientation at the end of a line. In other words, Figure 5a summarizes the effects of an "uncertainty principle" whereby "orientational certainty" along scenic edges implies "positional uncertainty" at line ends and corners. Stated in a vacuum, this breakdown does not seem to be particularly interesting. Stated in the shadow of the featural filling-in process, it has momentous implications. Without further processing that is capable of compensating for this breakdown, the BC System could not generate boundaries corresponding to scenic line ends and corners. Consequently, within the FC System, boundary signals would not exist at positions corresponding to line ends (Figure 6). The FC signals generated by the interior of each line could then initiate spreading of featural quality to perceptual regions beyond the location of the line end. In short, the failure of boundary detection at line ends could enable colors to flow out of every line end! In order to prevent this perceptual catastrophe, orientational tuning, just like discounting the illuminant, must be followed by a hierarchy of compensatory processing stages in order to gain full effectiveness.

To offset this breakdown under normal circumstances, we have hypothesized that outputs from the cells with oriented receptive fields input to two successive stages of competitive interaction (Grossberg, 1984a; Grossberg \& Mingolla, 1985a), which are described in greater detail in Section 20 and the Appendix. These stages are designed to compensate for orientational insensitivity at the ends of lines and corners. Figure $5 \mathrm{~b}$ shows how these competitive interactions generate horizontal BC signals at the end of a vertical line. These "illusory" boundary contours help to prevent the flow of featural contrast from 

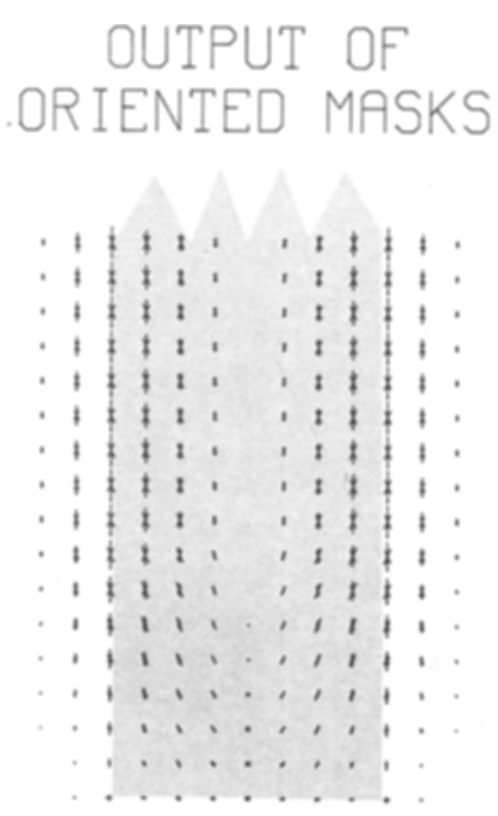

(a)
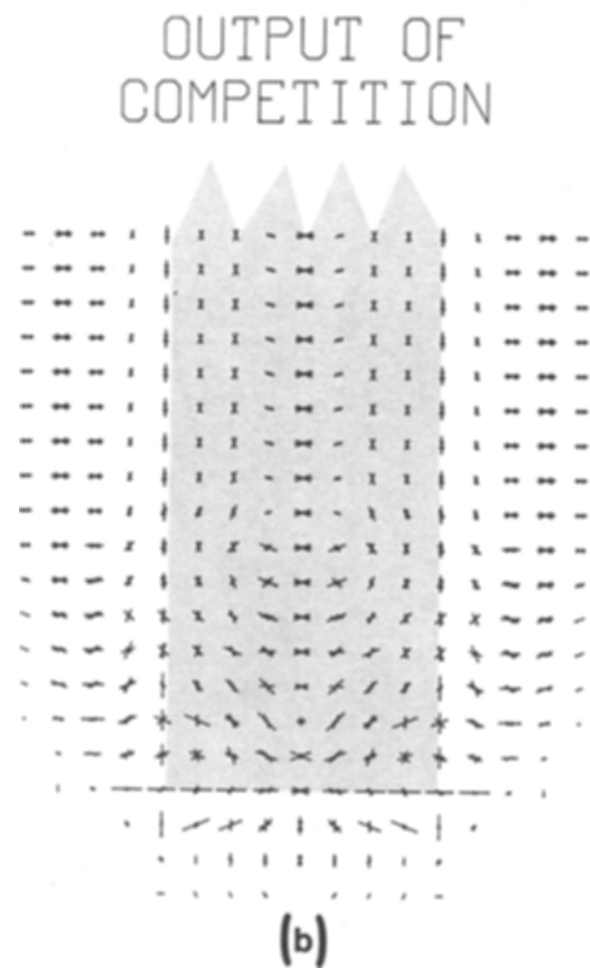

Figure 5. (a) An orientation field: Lengths and orientations of lines encode the relative sizes of the activations and orientations of the input masks at the corresponding positions. The input pattern, which is a vertical line end as seen by the receptive fields, corresponds to the shaded area. Fach mask has total exterior dimension of $16 \times 8$ units, with a unit length being the distance between two adjacent lattice positions. (b) Response of the potentials $y_{i j k}$ of the dipole field defined in the Appendix to the orientation field of Figure 5a: End cutting generates horizontal activations at line end locations that receive small and orientationally ambiguous input activations.

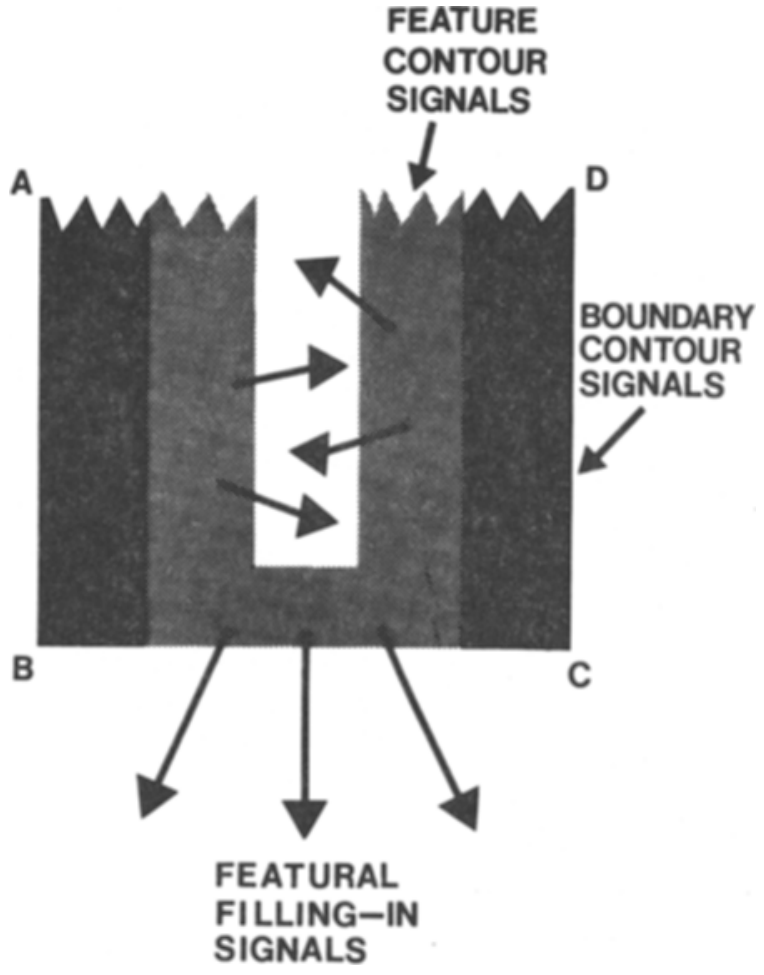

Figure 6. Possible spurious flow within the FC System of featural quality from line ends: Labels $A B C D$ outline the positions corresponding to the tip of a vertically oriented thin line. The black areas from $A$ to $B$ and from $C$ to $D$ indicate regions of the FC System which receive signals due to direct image-induced activation of vertically oriented receptive fields within the BC System. The stippled areas indicate regions of the FC System which receive FC signals from the interior of the line image. FC System receptive fields, being small and unoriented, may be excited at line ends, even if the oriented receptive fields of the BC System are not. The arrows indicate that filling-in due to these FC signals can spread outside the putative boundary ABCD of the line end.

the line end. Such horizontal boundary contours induced by a vertical line end are said to be generated by end cutting, or orthogonal induction.

The circle illusion that is perceived by a glance at Figure 7 can now be understood. The $\mathrm{BC}$ end cuts at the line ends can cooperate with other end cuts of similar orientation that are approximately aligned across perceptual space, just as boundary contours do to generate the percept of a Kanizsa square in Figure 3. These boundary contours group "illusory" figures for the same reason that they complete figures across retinal veins and blind spots. Within the BC System, both "real" and "illusory" contours are generated by the same dynamical laws.

\section{Parallel Induction by Edges Versus Perpendicular Induction by Line Ends}

Knowing the directions in which boundary contours will form is obviously essential to understanding perceptual grouping. Why does a boundary form parallel to the inducing edges in Figure 3 but perpendicular to the line ends in Figure 7? This is clearly a question about spatial scale, since thickening a line until its end becomes an edge will 

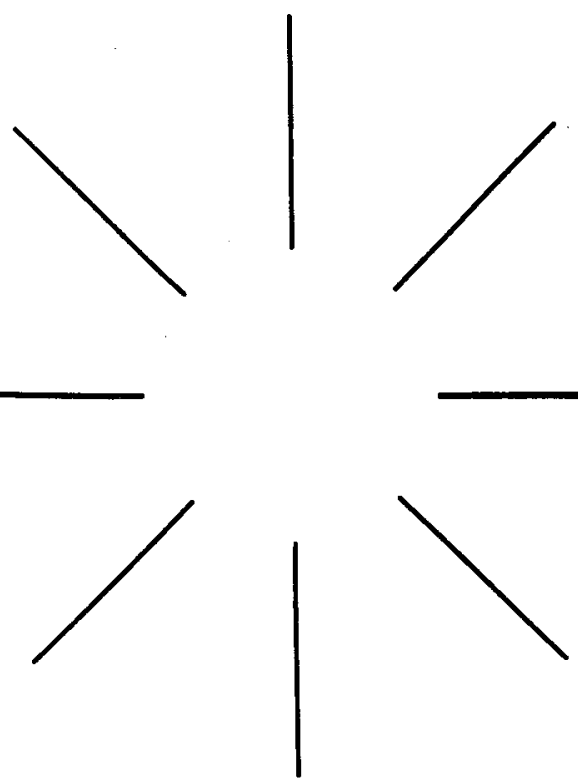

Figure 7. Cooperation among end-cut signals: $A$ bright illusory circle is induced perpendicular to the ends of the radial lines.

cause induction to switch from being perpendicular to the line to being parallel to the edge.

An answer to this question can be seen by inspection of Figure 5. In Figure 5a, strong vertical reactions occur in response to the long vertical edge of the line. Figure 5b shows that these vertical reactions remain vertical when they pass through the competitive stages. This is analogous to a parallel induction, since the vertical reactions in Figure 5b will generate a completed vertical boundary contour that is parallel to its corresponding scenic edge. By contrast, the ambiguous reaction at the line end in Figure 5a generates a horizontal end cut in Figure $5 b$ that is perpendicular to the line. If we thicken the line into a bar, it will eventually become wide enough to enable the horizontally oriented receptive fields at the bar end to generate strong reactions, in just the same way as the vertically oriented receptive fields along the side of the line generated strong vertical reactions there. The transition from ambiguous to strong horizontal reactions as the line end is thickened corresponds to the transition between perpendicular and parallel boundary contour induction.

This predicted transition has been discovered in electrophysiological recordings from cells in the monkey visual cortex (von der Heydt, Peterhans, \& Baumgartner, 1984). The pattern of cell responding in Figure $5 \mathrm{a}$ is similar to the data which von der Heydt et al. recorded in area 17 of the striate cortex, whereas the pattern of cell responding in Figure $5 \mathrm{~b}$ is similar to the data that von der Heydt et al. recorded in area 18 of the prestriate cortex. See Grossberg (1985) and Grossberg and Mingolla (1985a) for a further discussion of these and other supportive neural data.

\section{Boundary Completion via Cooperative-Competitive} Feedback Signaling: CC Loops and

the Statistics of Grouping

Another mechanism important in determining the directions in which perceptual groupings occur will now be summarized. As in Figure 5b, the outputs of the competitive stages can generate bands of oriented responses. These bands enable cells sensitive to similar orientations at approximately aligned positions to begin cooperating to form the final $\mathrm{BC}$ percept. These bands play a useful role, because they increase the probability that spatially separated $B C$ fragments will be aligned well enough to cooperate.

Figure 8 provides visible evidence of the existence of these bands. In Figure 8a, the end cuts that are exactly perpendicular to their inducing line ends can group to form a square boundary. In Figure $8 \mathrm{~b}$, the end cuts that are

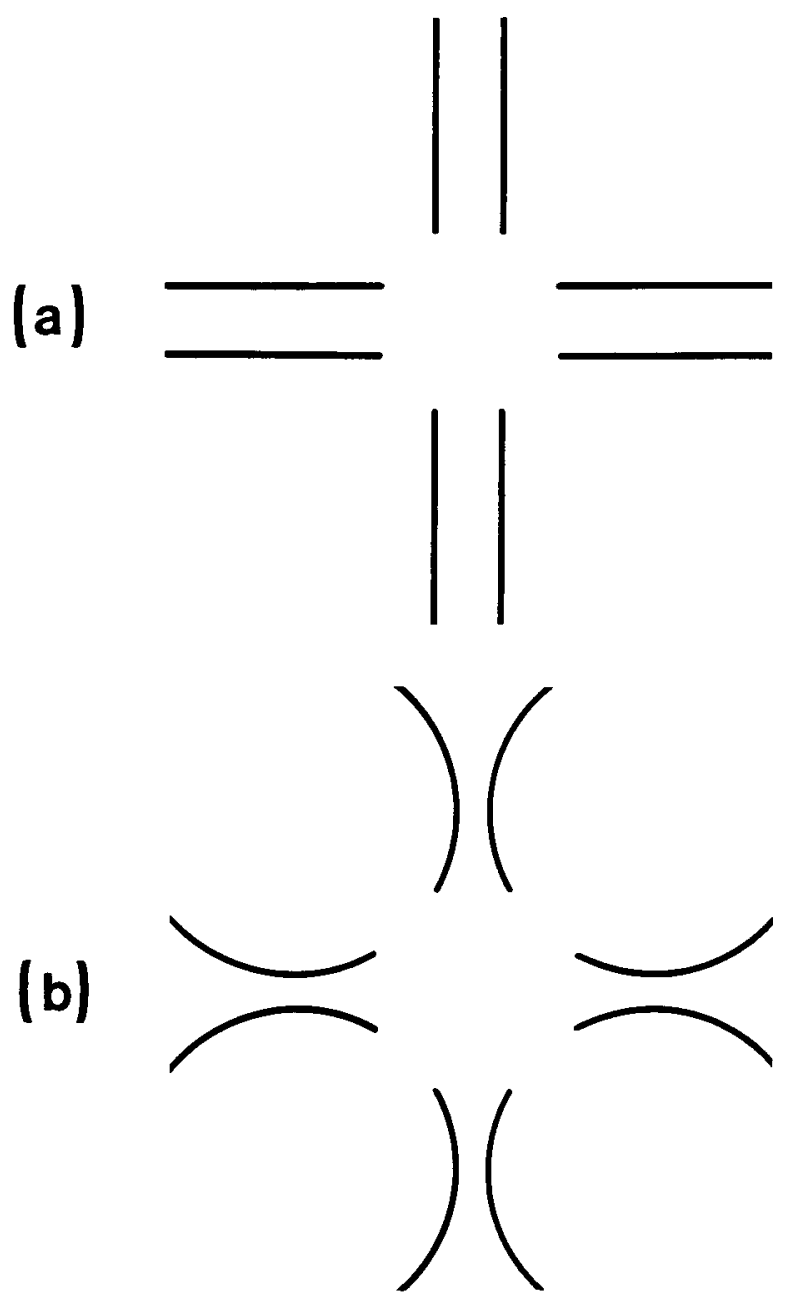

Figure 8. Evidence for bands of orientation responses: In (a), an illusory square is generated with sides perpendicular to the inducing lines. In (b), an illusory square is generated by lines with orientations that are not exactly perpendicular to the illusory contour. Redrawn from Kennedy (1979). 
exactly perpendicular to the line ends cannot group, but end cuts that are almost perpendicular to the line ends can.

Figure 8 also raises the following issue. If bands of end cuts exist at every line end, then why cannot all of them group to form bands of different orientations, which might sum to create fuzzy boundaries? How is a single sharp global boundary selected from among all of the possible local bands of orientations?

We suggest that this process is accomplished by the type of feedback exchange between competitive and cooperative processes that is depicted in Figure 9. We call such a competitive-cooperative feedback exchange a CC loop. Figure 9a shows that the competitive and cooperative processes occur at different network stages, with the competitive stage generating the end cuts depicted in Figure 5b. Thus, the outcome of the competitive stage serves as a source of inputs to the cooperative stage and receives feedback signals from the cooperative stage.

Each cell in the cooperative process can generate output signals only if it receives a sufficient number and intensity of inputs within both of its input-collecting branches. Thus, the cell acts like a type of logical gate,
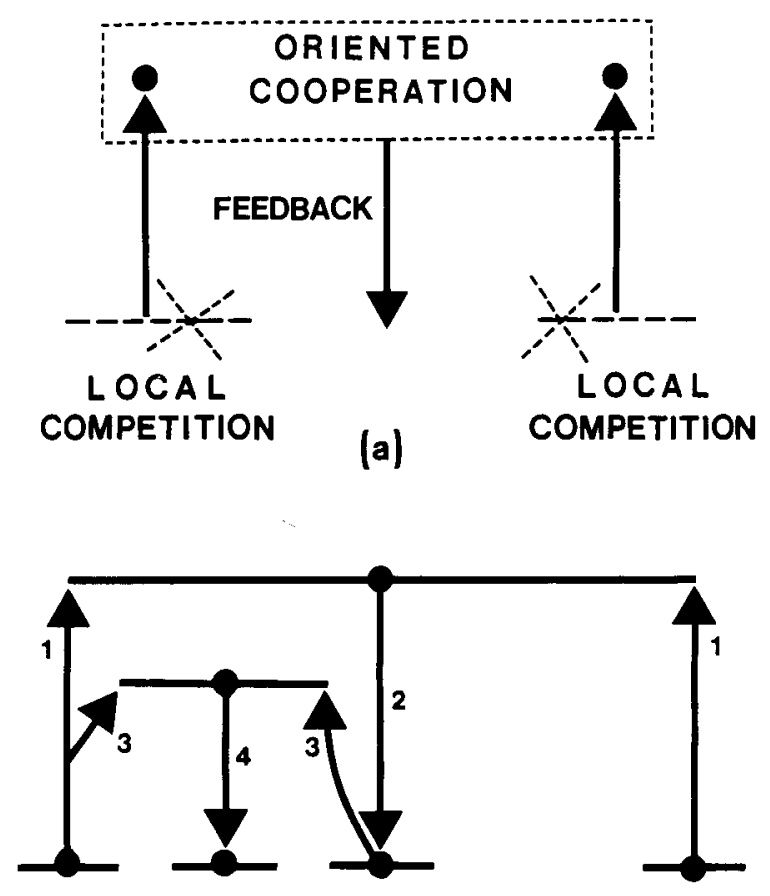

(b)

Figure 9. Boundary completion in a cooperative-competitive feedback exchange (CC loop): (a) Local competition occurs between different orientations at each spatial location. A cooperative boundary completion process can be activated by pairs of aligned orientations that survive their local competitions. This cooperative activation initiates the feedback to the competitive stage that is detailed in Figure 9b. (b) The pair of pathways 1 activate positive boundary completion feedback along pathway 2 . Then pathways such as 3 activate positive feedback along pathways such as 4 . Rapid completion of a sharp boundary between pathways 1 can hereby be generated. See text for details. or statistical dipole. The inputs to each branch come from cells of the competitive process that have an orientation and position that are similar to the spatial alignment of the cooperative cell's branches. When such a cell is activated, say by the conjoint action of both input pathways labeled 1 in Figure 9b, it sends excitatory feedback signals along the pathways labeled 2 . These feedback signals activate cells within the competitive stage which code a similar orientation and spatial position.

The cells at the competitive stage cannot distinguish whether they are activated by bottom-up signals from oriented receptive fields or by top-down signals from the cooperative stage. Either source of activation can cause them to generate bottom-up competitive-to-cooperative signals. Thus, new cells at the cooperative stage may now be activated by the conjoint action of both the input pathways labeled 3 in Figure $9 \mathrm{~b}$. These newly activated cooperative cells can then generate feedback signals along the pathway labeled 4 .

In this way, a rapid exchange of signals between the competitive and cooperative stages may occur. These signals can propagate inward between pairs of inducing BC inputs, as in the Kanizsa square of Figure 3, and can thereby complete boundaries across regions that receive no bottom-up inputs from oriented receptive fields. The process of boundary completion occurs discontinuously across space by using the gating properties of the cooperative cells (Figure $9 \mathrm{~b}$ ) to successively interpolate boundaries within progressively finer intervals. This type of boundary completion process is capable of generating sharp boundaries, with sharp endpoints, across large spatial domains (Grossberg \& Mingolla, 1985a). Unlike a low-spatial-frequency filter, the boundary-completion process does not sacrifice fine spatial resolution to achieve a broad spatial range.

Quite the contrary is true, since the CC loop sharpens, or contrast-enhances, the input patterns it receives from oriented receptive fields. This process of contrast enhancement is due to the fact that the cooperative stage feeds its excitatory signals back into the competitive stage. Thus, the competitive stage does double duty: it helps to complete line ends that oriented receptive fields cannot detect, and it helps to complete boundaries across regions that may receive no inputs whatsoever from oriented receptive fields. In particular, the excitatory signals from the cooperative stage enhance the competitive advantage of cells with the same orientation and position at the competitive stage (Figure 9b). As the competitive-cooperative feedback process unfolds rapidly through time, these local competitive advantages are synthesized into a global boundary grouping which can best reconcile all these local tendencies. In the most extreme version of this contrast-enhancement process, only one orientation at each position can survive the competition. That is, the network makes an orientational choice at each active position. The design of the CC loop is based upon theorems that characterize the factors that enable contrastenhancement and choices to occur within nonlinear cooperative-competitive feedback networks (Ellias \& 
Grossberg, 1975; Grossberg, 1973; Grossberg \& Levine, 1975).

As this choice process proceeds, it completes a boundary between some, but not all, of the similarly oriented and spatially aligned cells within the active bands of the competitive process (Figure 8). This interaction embodies a type of real-time statistical decision process whereby the most favorable groupings of cells at the competitive stage struggle to win over other possible groupings by initiating advantageous positive feedback from the cooperative stage. As Figure $8 \mathrm{~b}$ illustrates, the orientations of the grouping that finally wins is not determined entirely by local factors. This grouping reflects global cooperative interactions that can override the most highly favored local tendencies, in this case the strong perpendicular end cuts.

The experiments of von der Heydt et al. (1984) also reported the existence of area 18 cells that act like logical gates. These experiments therefore suggest that either the second stage of competition, or the cooperative stage, or both, occur within area 18 . Thus, although these BC System properties were originally derived from an analysis of perceptual data, they have successfully predicted recent neurophysiological data concerning the organization of mammalian prestriate cortex.

\section{Form Perception vs. Object Recognition: Invisible but Potent Boundaries}

One final remark needs to be made before turning to a consideration of textured scenes. Boundary contours in themselves are invisible. Boundary contours gain visibility by separating FC signals into two or more domains whose featural contrasts, after filling-in takes place, turn out to be different. (See Cohen \& Grossberg, 1984a, and Grossberg, 1985, for a discussion of how these and later stages of processing help to explain monocular and binocular brightness data.) We distinguish this role of boundary contours in generating visible form percepts from the role played by boundary contours in object recognition. We claim that completed BC signals project directly to the object-recognition system (Figure 1). Boundary contours thus need not be visible in order to strongly influence object recognition. An "illusory" BC grouping that is caused by a textured scene can have a much more powerful effect on scene recognition than the poor visibility of the grouping might indicate.

We also claim that the object-recognition system sends learned top-down template, or expectancy, signals back to the BC System (Carpenter \& Grossberg, 1985, in press; Grossberg, 1980, 1982, 1984b). Our theory hereby both agrees with and disagrees with the seminal idea of Gregory (1966) that "cognitive contours" are critical in boundary completion and object recognition. Our theory suggests that boundary contours are completed by a rapid, preattentive, automatic process as they activate the bottom-up adaptive filtering operations that activate the object-recognition system. The reaction within the objectrecognition system determines which top-down visual templates to the BC System will secondarily complete the BC grouping based upon learned "cognitive"' factors. These "doubly completed" boundary contours send signals to the FC System to determine the perceptual domains within which featural filling-in will take place.

We consider the most likely location of the boundary completion process to be area 18 (or V2) of the prestriate cortex (von der Heydt et al., 1984), the most likely location of the final stages of color and form perception to be area V4 of the prestriate cortex (Desimone, Schein, Moran, \& Ungerleider, 1985; Zeki, 1983a, 1983b), and the most likely location of some aspects of object recognition to be the inferotemporal cortex (Schwartz, Desimone, Albright, \& Gross, 1983). These anatomical interpretations were chosen after a comparison was made between theoretical properties and known neural data (Grossberg \& Mingolla, 1985a). They also provide markers for performing neurophysiological experiments to further test the theory's mechanistic predictions.

\section{Analysis of the Beck Theory of Textural Segmentation: Invisible Collinear Cooperation}

We now begin a dynamical explanation and refinement of the main properties of Beck's important theory of textural segmentation (Beck et al., 1983). One of the central hypotheses of the Beck theory is that "local linking operations form higher-order textural elements" (p. 2). "Textural elements are hypothesized to be formed by proximity, certain kinds of similarity, and good continuation. Others of the Gestalt rules of grouping may play a role in the formation of texture .... There is an encoding of the brightness, color, size, slope, and the location of each textural element and its parts"' (p. 31). We will show that the properties of these "textural elements" are remarkably similar to the properties of the completed boundaries that are formed by the BC System. To explain this insight, we will analyze various of the images used by Beck et al. in the light of BC System properties.

Figure 10 provides a simple example of what the Beck school means by a "textural element." Beck et al. (1983) write: "The short vertical lines are linked to form long lines. The length of the long lines is an "emergent feature' which makes them stand out from the surrounding short lines"' (p. 5). The linking per se is explained by our theory in terms of the process whereby similarly oriented and spatially aligned outputs from the second competitive stage can cooperate to complete a collinear intervening boundary contour.

One of the most remarkable aspects of this "emergent feature" is not analyzed by Beck et al. Why do we continue to see a series of short lines if long lines are the emergent features that control perceptual grouping? In our theory, the answer to this question is as follows. Within the BC System, a boundary structure emerges corresponding to the long lines described by Beck et al. This structure includes a long vertical component as well as short horizontal end cuts near the endpoints of the short scenic lines. The output of this BC structure to the FC System 


$\begin{array}{llllllllllllll}1 & 1 & 1 & 1 & 1 & 1 & 1 & 1 & 1 & 1 & 1 & 1 & 1 \\ 1 & 1 & 1 & 1 & 1 & 1 & 1 & 1 & 1 & 1 & 1 & 1 & 1 \\ 1 & 1 & 1 & 1 & 1 & 1 & 1 & 1 & 1 & 1 & 1 & 1 & 1 \\ 1 & 1 & 1 & 1 & 1 & 1 & 1 & 1 & 1 & 1 & 1 & 1 & 1 \\ 1 & 1 & 1 & 1 & 1 & 1 & 1 & 1 & 1 & 1 & 1 & 1 & 1 \\ 1 & 1 & 1 & 1 & 1 & 1 & 1 & 1 & 1 & 1 & 1 & 1 & 1 & 1 \\ 1 & 1 & 1 & 1 & 1 & 1 & 1 & 1 & 1 & 1 & 1 & 1 & 1 & 1 \\ 1 & 1 & 1 & 1 & 1 & 1 & 1 & 1 & 1 & 1 & 1 & 1 & 1 \\ 1 & 1 & 1 & 1 & 1 & 1 & 1 & 1 & 1 & 1 & 1 & 1 & 1 & 1 \\ 1 & 1 & 1 & 1 & 1 & 1 & 1 & 1 & 1 & 1 & 1 & 1 & 1 \\ 1 & 1 & 1 & 1 & 1 & 1 & 1 & 1 & 1 & 1 & 1 & 1 & 1 & 1 \\ 1 & 1 & 1 & 1 & 1 & 1 & 1 & 1 & 1 & & 1 & 1 & 1 & 1 \\ 1 & 1 & 1 & 1 & 1 & 1 & 1 & 1 & 1 & 1 & 1 & 1 & 1 & 1\end{array}$

Figure 10. Emergent features: The collinear linking of short line segments into longer segments is an "emergent feature" which sustains textural grouping. Our theory explains how such emergent features can contribute to perceptual grouping even if they are not visible. (Reprinted, by permission, from Beck, Prazdny, \& Rosenfeld, 1983.)

prevents featural filling-in of dark and light contrasts from crossing the boundaries corresponding to the short lines. On the other hand, the output from the BC System to the object-recognition system reads out a long-line structure without regard to which subsets of this structure will be perceived as dark or light.

This example points to a possible source of confusion in the Beck model. Beck et al. (1983) claim that "there is an encoding of the brightness, color, size, slope, and the location of each textural element and its parts" (p. 31). Figure 10 illustrates a sense in which this assertion is false. The long BC structure can have a powerful effect on textural segmentation even if it has only a minor effect on the brightness percepts corresponding to the short lines in the image, because an emergent boundary contour can generate a large input to the Object Recognition System (OR System) without generating a large brightness difference. The Beck model does not adequately distinguish between the contrast sensitivity that is needed to activate elongated receptive fields at an early stage of boundary formation and the effects of completed boundaries on featural filling-in. The outcome of featural fillingin, rather than the contrast sensitivity of the BC System's elongated receptive fields, helps to determine a brightness or color percept (Cohen \& Grossberg, 1984a; Grossberg \& Mingolla, 1985a).

A related source of ambiguity in the Beck model arises from the fact that the strength of an emergent boundary contour does not even depend on image contrasts, let alone brightness percepts, in a simple way. The Beck model does not adequately distinguish between the ability of elongated receptive fields to activate a boundary contour in regions where image contrast differences do exist and the cooperative interactions that complete the boundary contour in regions where image-contrast differences may or may not exist. The cooperative interaction may, for example, alter boundary contours at positions that lie within the receptive fields of the initiating orientation-sensitive cells, as in Figure 8b. The final percept, even at positions that directly receive image contrasts, may be strongly influenced by cooperative interactions that reach these positions by spanning positions that do not directly receive image contrasts. This property is particularly important in situations in which a spatial distribution of statistically determined image contrasts, such as dot or letter densities, form the image that excites the orientation-sensitive cells.

\section{The Primacy of Slope}

Figure 11 illustrates this type of interaction between bottom-up direct activation of orientationally tuned cells and top-down cooperative interaction of such cells. Beck and his colleagues have constructed many images of this type to demonstrate that orientation or "slope is the most important of the variables associated with shape for producing textural segmentation.... A tilted $\mathrm{T}$ is judged to be more similar to an upright $T$ than is an $L$. When these figures are repeated to form textures ... the texture made up of $\mathrm{Ls}$ is more similar to the texture made up of upright Ts than to the texture made up of tilted Ts" (Beck et al., 1983, p. 7). In our theory, this fact follows from several properties acting together: the elongated receptive fields in the BC System are orientationally tuned. This property provides the basis for the system's sensitivity to slope. As collinear boundary completion takes place due to cooperative-competitive feedback (Figure 9), it can group together approximately collinear boundary contours that arise from contrast differences due to the different letters. Collinear components of different letters are grouped just as the BC System groups image contrasts due to a single scenic edge that excites the retina on opposite sides of a retinal vein. The number and density of inducing elements of similar slope can influence the strength of the final set of boundary contours pointing in the same direction. Both Ls and Ts generate many horizontal and vertical boundary inductions, whereas tilted Ts generate diagonal boundary inductions.

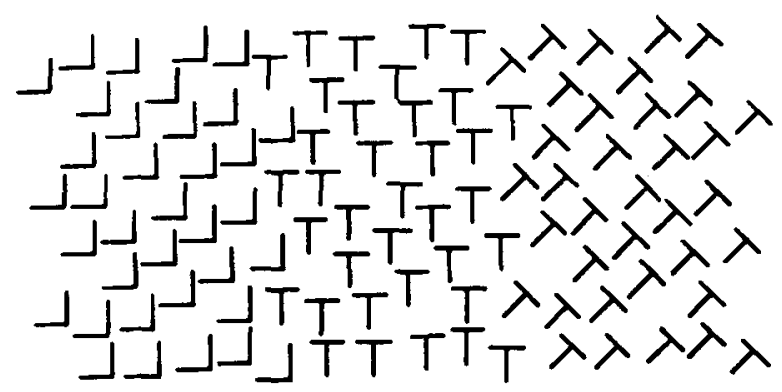

Figure 11. The primacy of slope: In this classic figure, textural segmentation between the tilted and upright $T s$ is far stronger than between the upright $T s$ and $\mathrm{Ls}$. The figure illustrates that grouping of disconnected segments of similar slope is a powerful basis for textural segmentation. (Reprinted, by permission, from Beck, Prazdny, \& Rosenfeld, 1983.) 
The main paradoxical issue underlying the percept of Figure 11 concerns how the visual system overrides the perceptually vivid individual letters. Once one understands mechanistically the difference between boundary completion and visibility, and the role of boundary completion in forming even individual edge segments without regard to their ultimate visibility, this paradox is resolved.

\section{Statistical Properties of Oriented Receptive Fields: OC Filters}

Variations on Figure 11 can also be understood by refining the above argument. In Beck (1966a), it is shown that Xs in a background of Ts produce weaker textural segmentation than a tilted $\mathrm{T}$ in a background of upright Ts, even though both images contain the same orientations. We agree with Beck et al. (1983) that "what is important is not the orientation of lines per se but whether the change in orientation causes feature detectors to be differentially stimulated" (p. 9). An X and a T have a centrally symmetric shape that weakens the activation of elongated receptive fields. A similar observation was made by Schatz (1977), who showed that changing the slope of a single line from vertical to diagonal led to stronger textural segmentation than changing the slope of three parallel lines from vertical to diagonal.

Both of these examples are compatible with the fact that orientationally tuned cells measure the statistical distribution of contrasts within their receptive fields. They do not respond only to a template of an edge, bar, or other definite image. They are sensitive to the relative contrast of light and dark on either side of their axis of preferred orientation (Appendix, Equation A1). Each receptive field at the first stage of boundary contour processing is divided into two halves along an oriented axis. Each half of the receptive field sums the image-induced inputs it receives. The integrated activation from one of the half-fields inhibits the integrated activation from the other half-field. A net output signal is generated by the cell if the net activation is sufficiently positive. This output signal grows with the size of the net activation. Thus, each such oriented cell is sensitive to amount of contrast (size of the net activation) and to direction of contrast (only one half-field inhibits the other half-field), in addition to being sensitive to factors such as orientation, position, and spatial frequency.

A pair of such oriented cells corresponding to the same position and orientation, but opposite directions of contrast, send converging excitatory pathways to cells defining the next stage in the network. These latter cells are therefore sensitive to factors such as orientation, position, spatial frequency, and amount of contrast, but they are insensitive to direction of contrast.

Together, the two successive stages of oriented cells define a filter that is sensitive to properties concerned with orientation and contrast. We therefore call this filter an $\mathrm{OC}$ filter. The OC filter inputs to the $\mathrm{CC}$ loop. The $\mathrm{BC}$ System network is a composite of $\mathrm{OC}$ filter and CC loop. The output cells of the OC filter, being insensitive to direction of contrast, are the ones that respond to the relative contrast of light and dark on either side of their axis of preferred orientation.

Both the Xs studied by Beck (1966a) and the multiple parallel lines studied by Schatz (1977) reduce this relative contrast. These images therefore weaken the relative and absolute sizes of the input to any particular orientation. Thus, even the "front end" of the BC System begins to regroup the spatial arrangement of contrast differences that is found within the scenic image.

\section{Competition between Perpendicular Subjective Contours}

A hallmark of the Beck approach has been the use of carefully chosen but simple figural elements in arrays whose spatial parameters can be easily manipulated. Arrays built up from $U$ shapes have provided a particularly rich source of information about textural grouping. In the bottom half of Figure 12, for example, the line ends of the Us and of the inverted Us line up in a horizontal direction. Their perpendicular end cuts can therefore cooperate, just as in Figures 7 and 8, to form long horizontal boundary contours. These long boundary contours enable the bottom half of the figure to be preattentively distinguished from the top half. Beck et al. (1983) note that segmentation of this image is controlled by "subjective contours" (p. 2). They do not use this phrase to analyze their other displays, possibly because the "subjective" boundary contours in other displays are not as visible.

The uncertainty within Beck et al. (1983) concerning the relationship between "linking operations" and "subjective contours" is illustrated by their analysis of Figure 13. In Figure 13a, vertical and diagonal lines alternate. In Figure 13b, horizontal and diagonal lines alternate. The middle third of Figure 13a is preattentively segmented better than the middle third of Figure 13b. Beck et al. (1983) explain this effect by saying that "the linking of the lines into chains also occurred more strongly

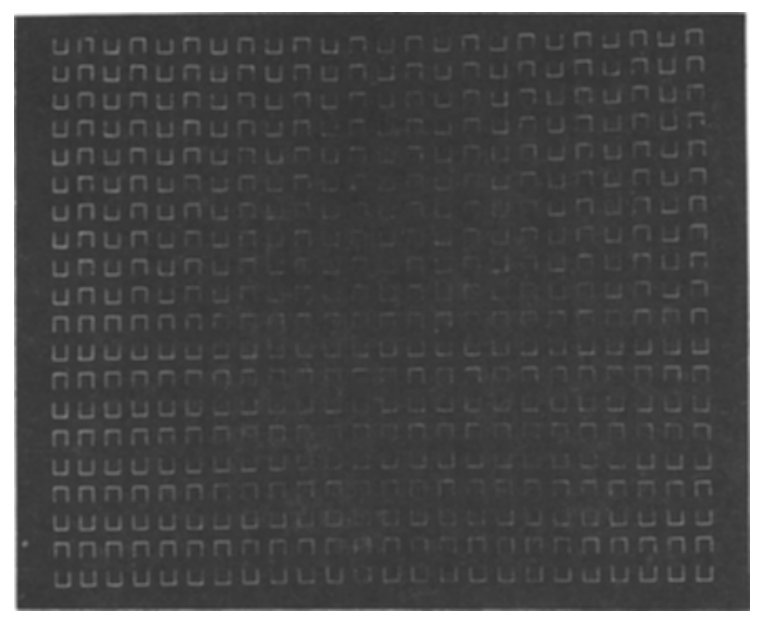

Figure 12. Textural grouping supported by subjective contours: Cooperation among end cuts generates horizontal subjective contours in the bottom half of this figure. (Reprinted, by permission, from Beck, Prazdny, \& Rosenfeld, 1983.) 


\begin{tabular}{|c|c|c|c|c|c|c|c|c|c|c|c|c|c|c|c|c|}
\hline & I & r & 1 & ' & 1 & r & 1 & ' & 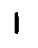 & r & 1 & $\prime$ & 1 & 2 & & \\
\hline & 1 & ' & 1 & ' & 1 & $\prime$ & 1 & ' & 1 & $\prime$ & 1 & 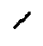 & I & ' & 1 & \\
\hline & 1 & ' & 1 & ' & 1 & ' & 1 & $\gamma$ & I & 1 & 1 & 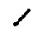 & I & $\gamma$ & 1 & \\
\hline & 1 & I & I & $\prime$ & 1 & $\prime$ & 1 & $\prime$ & 1 & $\prime$ & I & 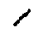 & I & $r$ & 1 & \\
\hline & 1 & ' & 1 & I & I & $\prime$ & 1 & , & I & $\prime$ & 1 & 1 & I & 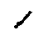 & 1 & \\
\hline & 1 & r & 1 & 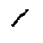 & I & $\prime$ & 1 & $r$ & & $r$ & 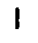 & 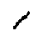 & I & $r$ & 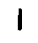 & \\
\hline & $\prime$ & 1 & , & 1 & 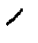 & 1 & $\prime$ & 1 & 1 & 1 & 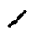 & 1 & ' & I & 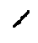 & \\
\hline & I & $\prime$ & 1 & $\prime$ & 1 & $\prime$ & 1 & 1 & 1 & $\prime$ & 1 & 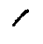 & 1 & $r$ & 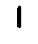 & \\
\hline & $\prime$ & 1 & $\prime$ & 1 & $\gamma$ & 1 & $\prime$ & 1 & 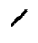 & 1 & 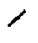 & 1 & $\gamma$ & I & 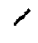 & \\
\hline & 1 & $\gamma$ & 1 & $\gamma$ & I & $\prime$ & 1 & $\gamma$ & 1 & $\gamma$ & 1 & $\gamma$ & 1 & $\gamma$ & 1 & \\
\hline & $\prime$ & I & 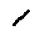 & 1 & 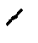 & 1 & ' & I & 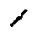 & 1 & 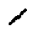 & 1 & 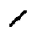 & 1 & ' & \\
\hline & 1 & $\prime$ & 1 & $\prime$ & 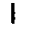 & $\prime$ & 1 & $\prime$ & 1 & $\prime$ & 1 & $\prime$ & 1 & $\prime$ & 1 & \\
\hline & 1 & ' & 1 & $\prime$ & 1 & $\prime$ & 1 & $\prime$ & 1 & $\nearrow$ & 1 & $\prime$ & 1 & $\prime$ & 1 & \\
\hline & 1 & $\prime$ & 1 & ' & 1 & ' & I. & $\prime$ & I & $\alpha$ & 1 & r & 1 & I & 1 & \\
\hline & 1 & I & 1 & $\prime$ & 1 & $\prime$ & 1 & $\prime$ & & $\prime$ & 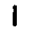 & 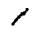 & 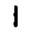 & ' & 1 & \\
\hline & 1 & - & 1 & $\gamma$ & 1 & $\prime$ & 1 & $\prime$ & & ' & 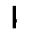 & 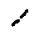 & 1 & ' & 1 & \\
\hline & 1 & r & 1 & $r$ & 1 & 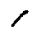 & 1 & $r$ & 1 & $\prime$ & 1 & 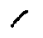 & 1 & 1 & 1 & \\
\hline
\end{tabular}

(a)

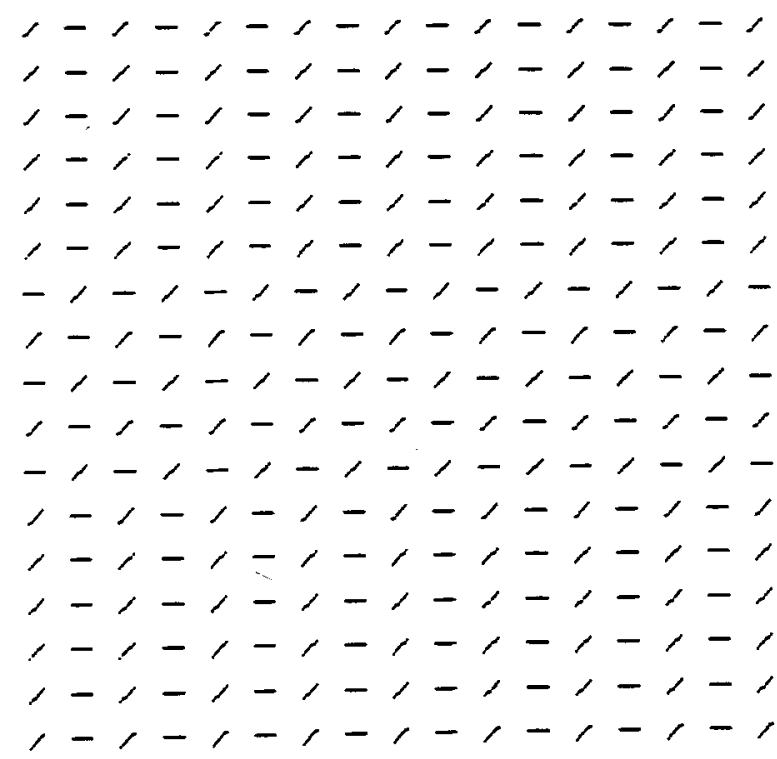

(b)

Figure 13. Effects of distance, perpendicular orientations, and collinearity on perceptual grouping: In both (a) and (b), vertical and horizontal subjective boundaries are generated. The text explains how the groupings in (a) better segregate the middle third of the figure. (Reprinted, by permission, from Beck, Prazdny, \& Rosenfeld, 1983.)

when the lines were collinear than when they were parallel, i.e., the linking of horizontal lines to form vertical columns"' (p. 21). "The horizontal lines tend to link in the direction in which they point. The linking into long horizontal lines competes with the linking of the lines into vertical columns and interferes with textural segmentation" (p. 22).
Our theory supports the spirit of this analysis. Both the direct outputs from horizontally oriented receptive fields and the vertical end cuts induced by competitive processing at horizontal line ends can feed into the collinear boundary completion process. The boundary completion process, in turn, feeds its signals back to a competitive stage where perpendicular orientations compete (Figure 9). Hence, direct horizontal activations and indirect vertical end cuts can compete at positions that receive both influences due to cooperative feedback.

Beck et al. (1983) do not, however, comment upon an important difference between Figures $13 \mathrm{a}$ and $13 \mathrm{~b}$ that is noticed when one realizes that linking operations may generate both visible and invisible subjective contours. We claim that, in Figure 13b, the end cuts of horizontal and diagonal line ends can cooperate to form long vertical boundary contours that run from the top to the bottom of the figure. As in Figure $8 \mathrm{~b}$, global cooperative factors can override local orientational preferences to choose end cuts that are not perpendicular to their inducing line ends. We suggest that this happens with respect to the diagonal line ends in Figure 13b due to the cooperative influence of the vertical end cuts that are generated by collinear horizontal line ends. The long vertical boundary contours that are hereby generated interfere with textural segmentation by passing through the entire figure.

This observation, by itself, is not enough to explain the better segmentation of Figure 13a. Due to the horizontal alignment of vertical and diagonal line ends in Figure 13a, horizontal boundary contours could cross this entire figure. In Figure 13a, however, vertical lines within the top and bottom thirds of the picture are contiguous to other vertical lines. In Figure 13b, diagonal lines are juxtaposed between every pair of horizontal lines. Thus, in Figure 13a, a strong tendency exists to form vertical boundary contours in the top and bottom thirds of the picture due both to the distance dependence of collinear cooperation and to the absence of competing intervening orientations. These strong vertical boundary contours can successfully compete with the tendency to form horizontal boundary contours that cross the figure. In Figure 13b, the tendencies to form vertical and horizontal boundary contours are more uniformly distributed across the figure. Thus, the disadvantage of Figure 13b may not just be due to the "linking into long horizontal lines [which] competes with the linking of the lines into vertical columns," as Beck et al. (1983, p. 22) suggest. We suggest that, even in Figure 13a, strong competition from horizontal linkages occurs throughout the figure. These horizontal linkages do not prevent preattentive grouping, because strong vertical linkages exist at the top and bottom thirds of the figure and these vertical groupings cannot bridge the middle third of the figure. In Figure $13 \mathrm{~b}$, by contrast, the competing horizontal linkages in the top and bottom third of the figure are weaker than they are in Figure 13a. Despite this, the relative strengths of emerging groupings corresponding to different parts of a scene, rather than the strengths of oriented activations at individual scenic 
positions, determine how well a region of the scene can be segmented.

\section{Multiple Distance-Dependent Boundary Contour Interactions: Explaining Gestalt Rules}

Figure 14 illustrates how changing the spatial separation of figural elements without changing their relative positions can alter interaction strengths at different stages of the BC System; different rearrangements of the same scenic elements can differentially probe the hierarchical organization of boundary processing. This type of insight leads us to suggest how different Gestalt rules are realized by a unified system of BC System interactions.

In the top half of Figure 14a, horizontal boundary contours that cross the entire figure are generated by horizontal end cuts at the tips of the inverted Us. These long boundary contours help to segregate the top half of the figure from its bottom, just as they do in Figure 12. This figure thus reaffirms that collinear cooperative interactions can span a broad spatial range. Some horizontal BC formation may also be caused by cooperation between the bottoms of the Us. We consider this process to be weaker in Figure 14a for the same reason that it is weaker in Figure 12: the vertical sides of the Us weaken it via competition between perpendicular orientations. Beck et al. (1983, p. 23), by contrast, assert that "the bottom lines of the U's link on the basis of colinearity (a special case of good continuation)," and say nothing about the horizontal boundary contours induced by the horizontal end cuts.

In Figure 14b, the $U$ and inverted- $U$ images are placed more closely together without otherwise changing their relative spatial arrangement. End cuts at the tips of the inverted Us again induce horizontal boundary contours across the top half of the figure. New types of grouping are also induced by this change in the density of the Us. The nature of these new groupings can most easily be understood by considering the bottom of Figure 14b. At a suitable viewing distance, one can now see diagonal groupings that run at $45^{\circ}$ and $135^{\circ}$ angles through the bases of the Us and inverted Us. We claim that these diagonal groupings are initiated when the density gets sufficiently high to enable diagonally oriented receptive fields to record relatively large image contrasts. In other words, at a low density of scenic elements, orientationally tuned receptive fields can be stimulated only by one $U$ or inverted $U$ at a time. At a sufficiently high density of scenic elements, each receptive field can be stimulated by parts of different scenic elements that fall within that receptive field. Once the diagonal receptive fields get activated, they can trigger diagonally oriented boundary completions. A similar possibility holds in the top half of Figure 14b. Horizontally and vertically tuned receptive fields can begin to be excited by more than one $U$ or inverted $U$. Thus, the transition from Figure 14a to Figure 14b preserves long-range horizontal cooperation based on competitive end cuts and other collinear horizontal interactions, and enables the earlier stage of oriented receptive fields to create new scenic groupings, notably in diagonal directions.

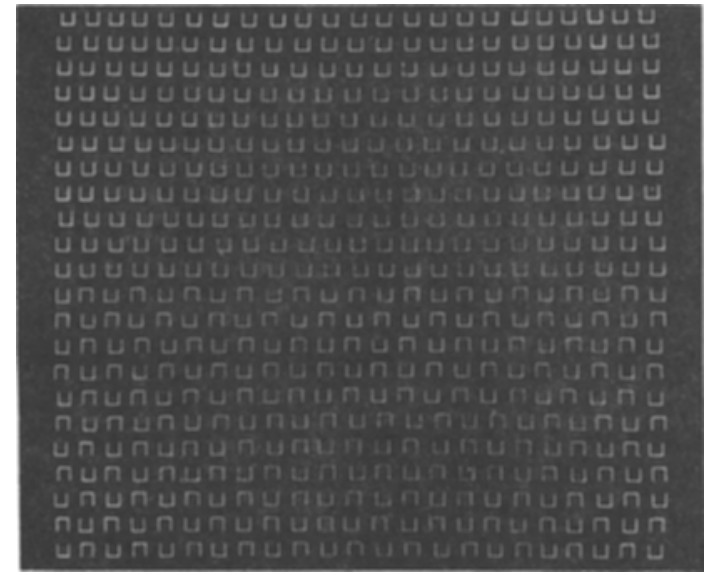

(a)

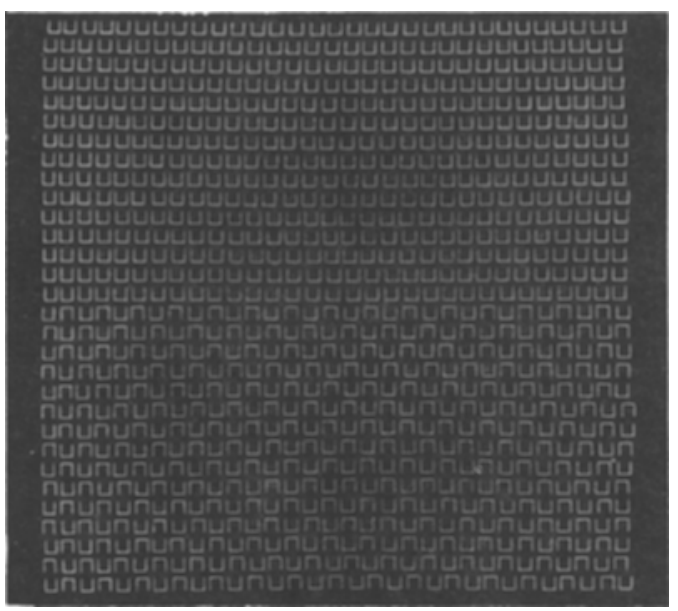

(b)

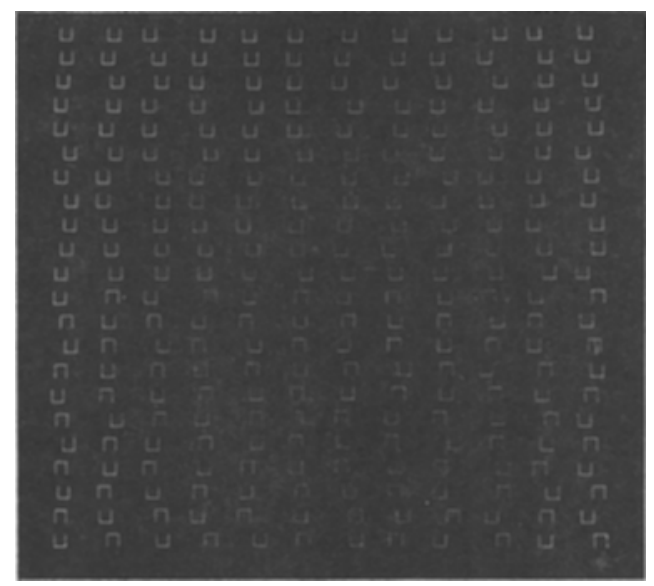

(c)

Figure 14. The importance of spatial scale: These three figures probe the subtle effects on textural grouping of varying spatial scale. For example, the diagonal grouping at the bottom of (b) is initiated by differential activation of diagonally oriented masks, despite the absence of any diagonal edges in the image. See the text for extended discussion. (Reprinted, by permission, from Beck, Prazdny, \& Rosenfeld, 1983.) 
Beck et al. (1983) analyze Figures 14a and 14b using Gestalt terminology. They say that segmentation in Figure 14a is due to "linking based on the colinearity of the base lines of the Us" (p. 24). Segmentation in Figure $14 \mathrm{~b}$ is attributed to "linking based on closure and good continuation" (p. 25). We suggest that both segmentations are due to the same BC System interactions, but that the scale change in Figure 14b enables oriented receptive fields and cooperative interactions to respond to new local groupings of image components.

In Figure 14c, the relative positions of Us and inverted Us are again preserved, but they are arranged to be closer together in the vertical than in the horizontal direction. These new columnar relationships prevent the image from segmenting into top and bottom halves. Beck et al. (1983) write that "strong vertical linking based on proximity interferes with textural segmentation"' (p. 28). We agree with this emphasis on proximity, but prefer a description which emphasizes that the vertical linking process uses the same textural segmentation mechanisms as are needed to explain all of their displays. We attribute the strong vertical linking to the interaction of five effects within the BC System. The higher relative density of vertically arranged Us and inverted Us provides a relatively strong activation of vertically oriented receptive fields. The higher density and stronger activation of vertically oriented receptive fields generates larger inputs to the vertically oriented long-range cooperative process, which enhances the vertical advantage by generating strong topdown positive feedback. The smaller relative density of horizontally arranged Us and inverted Us provides a relatively weak activation of horizontally oriented receptive fields. The lower density and smaller activation of these horizontally oriented receptive fields generates a smaller input to the horizontally oriented cooperative process. The horizontally oriented cooperation consequently cannot offset the strength of the vertically oriented cooperation. Although the horizontal end cuts can be generated by individual line ends, the reduction in density of these line ends in the horizontal direction reduces the total input to the corresponding horizontally oriented cooperative cells. All of these factors favor the ultimate dominance of vertically oriented long-range $\mathrm{BC}$ structures.

Beck et al. (1983) analyze the different figures in Figure 14 using different combinations of classical Gestalt rules. We analyze these figures by showing how they differentially stimulate the same set of BC System rules. This type of mechanistic synthesis leads to the suggestion that the BC System embodies a universal set of rules for textural grouping.

\section{Image Contrasts and Neon Color Spreading}

Beck et al. (1983) used regular arrays of black and gray squares on a white background and of white and gray squares on a black background with the same incisiveness as they used $U$ displays. All of the corresponding perceptual groupings can be qualitatively explained in terms of the contrast sensitivity of BC System responses to these images. The most difficult new property of these percepts can be seen by looking at Figure 15. Diagonal gray bands can be seen joining the gray squares in the middle third of the figure. We interpret this effect to be a type of neon color spreading (van Tuijl, 1975). This interpretation is supported by the percept that obtains when the gray squares are replaced by red squares of similar contrast, as we have done using our computer graphics system. Then diagonal red bands can be seen joining the red squares in the middle of the figure. Neither these red diagonal bands nor, by extension, the gray bands seen upon inspection of Figure 15, can be interpreted as being merely a classical contrast effect due to the black squares.

The percept of these diagonal bands can be explained using the same type of analysis that Grossberg (1984a) and Grossberg and Mingolla (1985a) have used to explain the neon color spreading that is induced by a black Ehrenstein figure surrounding a red cross (Figure 16; Redies \& Spillmann, 1981) and the complementary color induction and spreading that is induced when parts of an image grating are achromatic and complementary parts are colored (van Tuijl, 1975). These explanations indicate how segmentation within the BC System can sometimes induce visible contrasts at locations where no luminance contrasts exist in the scenic image.

Neon spreading phenomena occur only when some scenic elements have greater relative contrasts with respect to the background than do the complementary scenic elements (van Tuijl \& de Weert, 1979). This prerequisite is satisfied by Figure 15 . The black squares are much more contrastive relative to the white ground than are the gray squares. Thus, the black-to-white contrasts can ex-

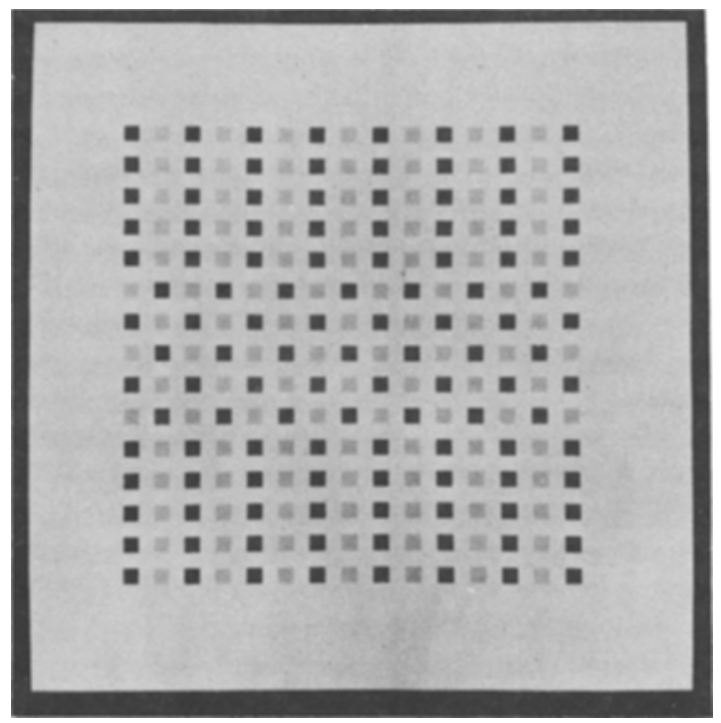

Figure 15. Textural segmentation and neon color spreading: The middle third of this figure is easily segmented from the rest. Diagonal flow of gray featural quality between the gray squares of the middle segment is an example of neon color spreading. See also Figures 16 and 17. (Reprinted from Beck, Prazdny, \& Rosenfeld, 1983. We are grateful to Jacob Beck for providing the original of this figure.) 


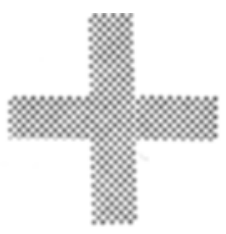

\section{(a)}

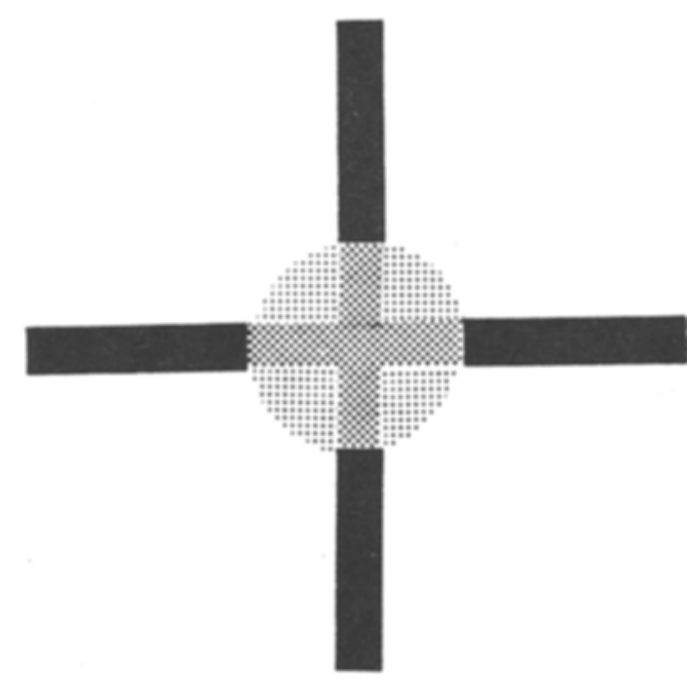

(b)

Figure 16. Neon color spreading: (a) A red cross in isolation appears unremarkable. (b) When the cross is surrounded by an Ehrenstein figure, the red color can flow out of the cross until it hits the illusory contour induced by the Ehrenstein figure.

cite oriented receptive fields within the BC System much more than can the gray-to-white contrasts. As in our other explanations of neon color spreading, we trace the initiation of this neon effect to two properties of the BC System: the contrast-sensitivity of the oriented receptive fields and the lateral inhibition within the first competitive stage among like-oriented cells at nearby positions (Section 20 and Appendix). Due to contrast sensitivity, each light-gray square activates oriented receptive fields less than does each black square. The activated orientations are, by and large, vertical and horizontal, at least on a sufficiently small spatial scale. At the first competitive stage, each strongly activated vertically tuned cell inhibits nearby weakly activated vertically tuned cells, and each strongly activated horizontally tuned cell inhibits nearby weakly activated horizontally tuned cells (Figure 17).

In all, each light-gray square's boundary contours receive strong inhibition both from the vertical and the horizontal direction. This conjoint vertical and horizontal inhibition generates a gap within the boundary contours at each corner of every light-gray square and a net tendency to generate a diagonal boundary contour via disinhibition at the second competitive stage. These diagonal boundary contours can then link up via collinear cooperation to further weaken the vertical and horizontal boundary contours as they build completed diagonal boundary contours between the light-gray squares. This lattice of diagonal boundary contours enables gray featural quality to flow out of the squares and fill in the positions bounded by the lattice within the FC System. In the top and bottom thirds of Figure 15, on the other hand, only the horizontal boundary contours of the gray squares are significantly inhibited. Such inhibitions tend to be compensated at the cooperative stage by collinear horizontal boundary completion. Thus, the integrity of the horizontal boundary contours near such a gray square's corner tends to be preserved.

It is worth emphasizing a similarity and a difference between the percepts in Figures 14b and 15. In both percepts, diagonal boundary contours help to segment the images. However, in Figure 14b, the diagonals are activated directly at the stage of the oriented receptive fields, whereas in Figure 15, the diagonals are activated indirectly via disinhibition at the second competitive stage. We suggest that similar global factors may partially determine the Hermann grid illusion. Spillmann (1985) has reviewed evidence that suggests a role for central factors in generating this illusion, notably the work of Preyer (1897/1898) and Prandtl (1927) showing that when a white

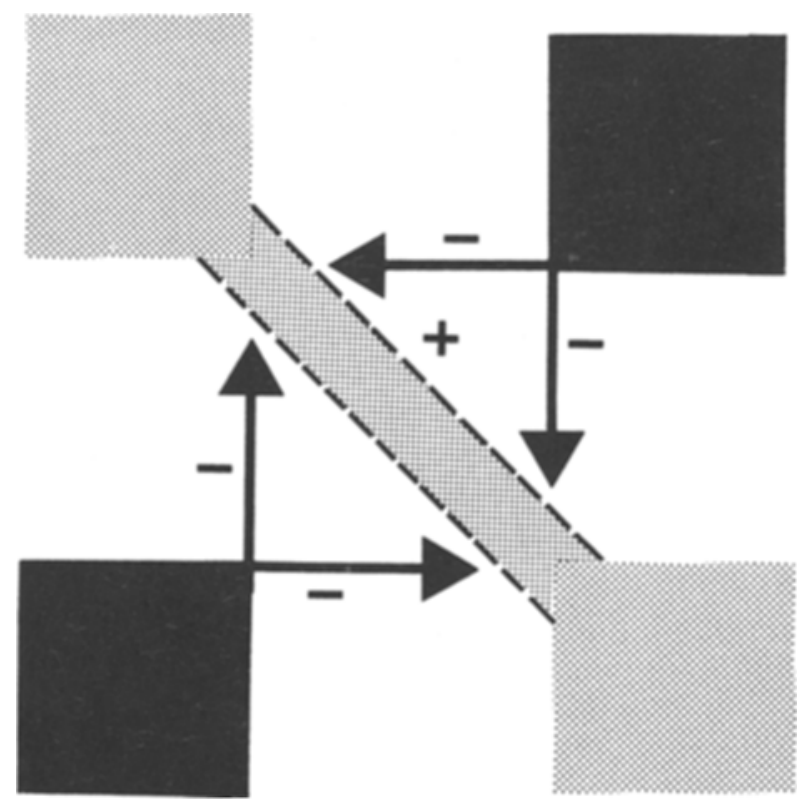

Figure 17. Boundary contour disinhibition and neon color spreading: This figure illustrates how the neon spreading evident in Figure 16 can occur. If gray squares are much lighter than black squares and the squares are sufficiently close, the net effect of strong inhibitory boundary signals from the black squares to the weakly activated gray square boundaries leads to disinhibition of diagonal boundary contours. Cooperation between these diagonal boundaries enables diagonal featural flow to occur between the gray squares. 
grid is presented on a colored background, the illusory spots have the same color as the surrounding squares. Wolfe (1984) has presented additional evidence that global factors contribute to this illusion.

Although we expect our theory to be progressively refined as it achieves a greater behavioral and neural explanatory range, we believe that the types of explanation suggested above will continue to integrate the several classical Gestaltist laws into a unified neo-Gestaltist mechanistic understanding. In this new framework, instead of invoking different Gestalt laws to explain different percepts, one analyses how different images probe the same laws in context-sensitive ways.

\section{Computer Simulations of Perceptual Grouping}

In this section, we summarize computer simulations that illustrate the BC System's ability to generate perceptual groupings akin to those in the Beck et al. displays. In the light of these results, we then analyze data of Glass and Switkes (1976) about random-dot percepts and of Gregory and Heard (1979) about border locking during the café wall illusion before defining rigorously the model neuron interactions that define the BC System.

Numerical parameters were held fixed for all of the simulations; only the input patterns were varied. As the input patterns were moved about, the BC System sensed relationships among the inducing elements and generated emergent boundary groupings among them. In all of the simulations, we defined the input patterns to be the output patterns of the oriented receptive fields, as in Figure 18a, since our primary objective was to study the CC loop, or cooperative-competitive feedback exchange. This step reduced the computer time needed to generate the simulations. If the BC System is ever realized in parallel hardware, rather than by simulation on a traditional computer, it will run in real time. In Figures 1825 , we have displayed network activities after the CC loop converges to an equilibrium state. These simulations used only a single cooperative bandwidth. They thus illustrate how well the BC System can segment images using a single "spatial frequency" scale. Multiple scales are, however, needed to generate three-dimensional form percepts (Grossberg, 1983b, 1985; Grossberg \& Mingolla, 1985b).

Figure 18a depicts an array of four vertically oriented input clusters. We call each cluster a Line because it represents a caricature of an orientation field's response to a vertical line (Figure 5a). In Figures $18 b, 18 c$, and $18 \mathrm{~d}$, we display the equilibrium activities of the cells at three successive $\mathrm{CC}$ loop stages: the first competitive stage, the second competitive stage, and the cooperative stage. The length of an oriented line at each position is proportional to the equilibrium activity of a cell whose receptive field is centered at that position with the prescribed orientation. We will focus upon the activity pattern within the y field, or second competitive stage,

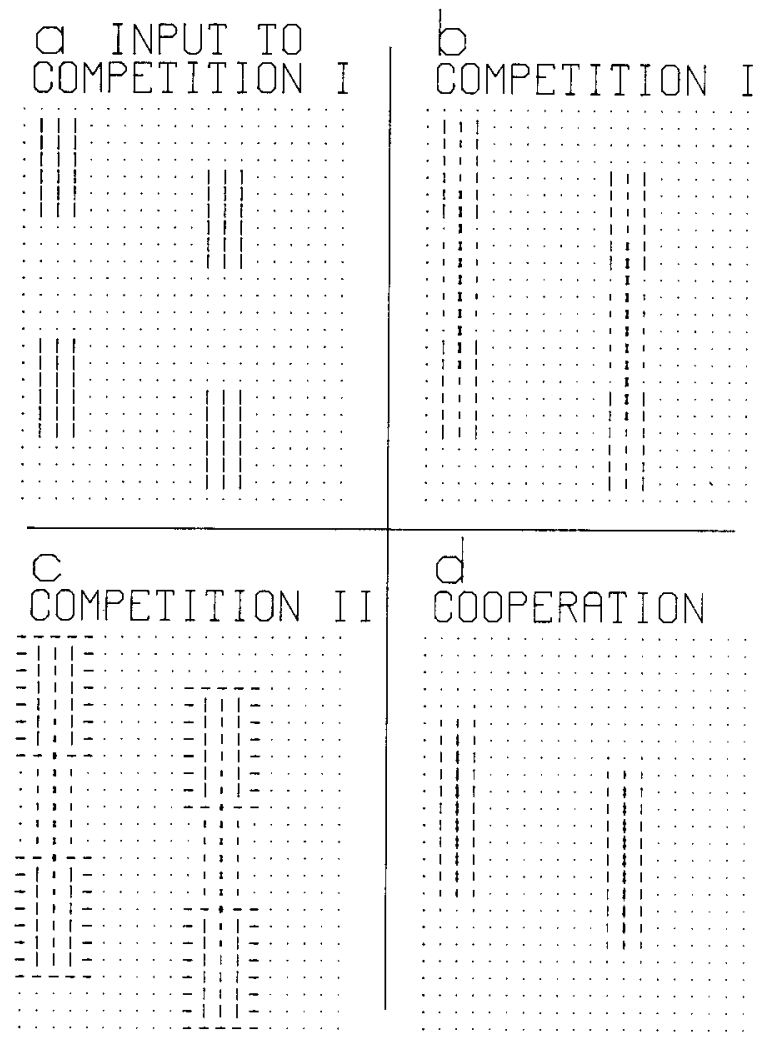

Figure 18. Computer simulation of processes underlying textural grouping: The length of each line segment in this figure and Figures 19-25 is proportional to the activation of a network node responsive to one of 12 possible orientations. The dots indicate the positions of inactive cells. In Figures 18-25, part (a) displays the results of input masks which sense the amount of contrast at a given orientation of visual input, as in Figure 5a. Parts (b)-(d) show equilibrium activities of oriented cells at the competitive and cooperative layers. A comparison of (a) and (c) indicates the major groupings sensed by the network. Here only the vertical alignment of the two left and two right Lines is registered. See text for detailed discussion.

of each simulation (Figure 18c). This is the final competitive stage that inputs to the cooperative stage (Section 8). The w-field (first competitive stage) and $z$-field (cooperative stage) activity patterns are also displayed to enable the reader to achieve a better intuition after considering the definitions of these fields in Section 20 and the Appendix.

The input pattern in Figure 18a possesses a manifest vertical symmetry: Pairs of vertical Lines are collinear in the vertical direction, whereas they are spatially outof-phase in the horizontal direction. The BC System senses this vertical symmetry, and generates emergent vertical lines in Figure 18c, in addition to horizontal end cuts at the ends of each Line, as suggested by Figure 10.

In Figure 19a, the input pattern shown in Figure 18a has been altered, so that the first column of vertical Lines is moved downward relative to the second column of vertical Lines. Figure 19c shows that the BC System begins 
to sense the horizontal symmetry within the input configuration. In addition to the emergent vertical grouping and horizontal end cuts like those of Figure 18c, an approximately horizontal grouping has appeared.

In Figure 20, the input Lines are moved so that pairs of Lines are collinear in the vertical direction and their Line ends are lined up in the horizontal direction. Now both vertical and horizontal groupings are generated in Figure 20c, as in Figure 13.

In Figure 21a, the input Lines are shifted so that they become noncollinear in a vertical direction, but pairs of their Line ends remain aligned. The vertical symmetry of Figure 20a is hereby broken. Thus, in Figure 21c, the BC System groups the horizontal Line ends, but not the vertical Lines.

Figure 22 depicts a more demanding phenomenon: the emergence of diagonal groupings where no diagonals whatsoever exist in the input pattern. Figure 22a is generated by bringing the two horizontal rows of vertical Lines closer together until their ends lie within the spatial bandwidth of the cooperative interaction. Figure $22 \mathrm{c}$ shows that the BC System senses diagonal groupings of the Lines, as in Figure 14b. It is remarkable that these di-

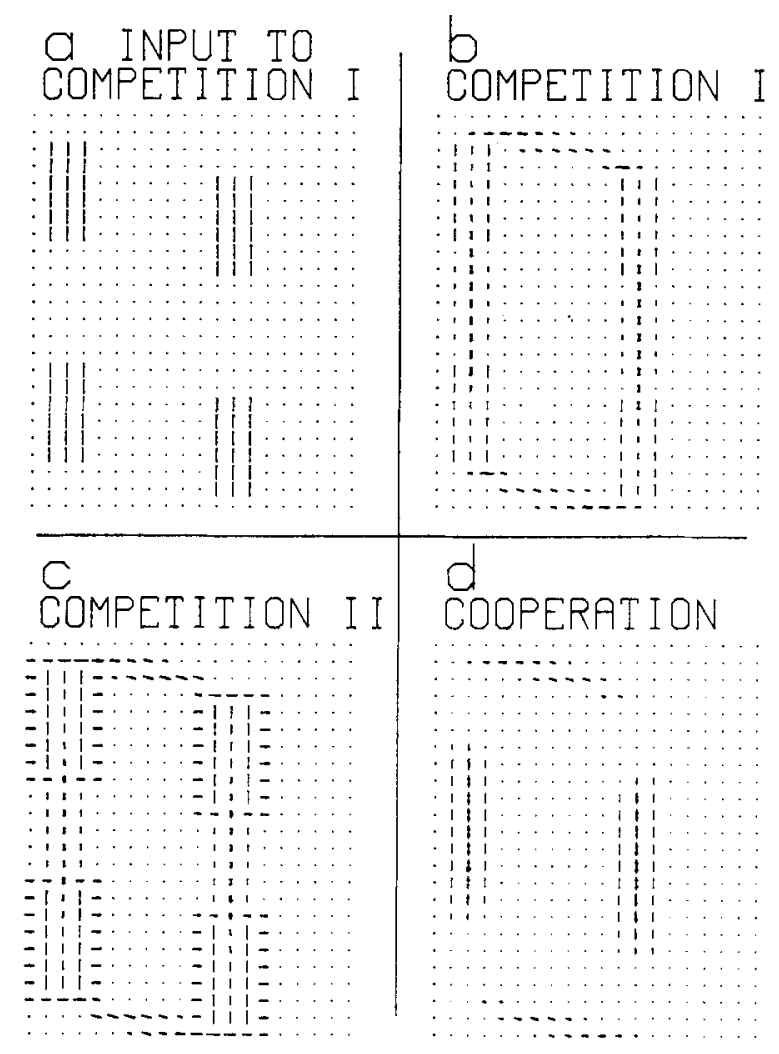

Figure 19. The emergence of nearly horizontal grouping: The only difference between the input for this figure and that of Figure 18 is that the left column of Lines has been moved downward by one lattice location. The vertical grouping of Figure 18 is preserved as the horizontal grouping emerges. The horizontal groupings are due to cooperation between end cuts at the Line ends.

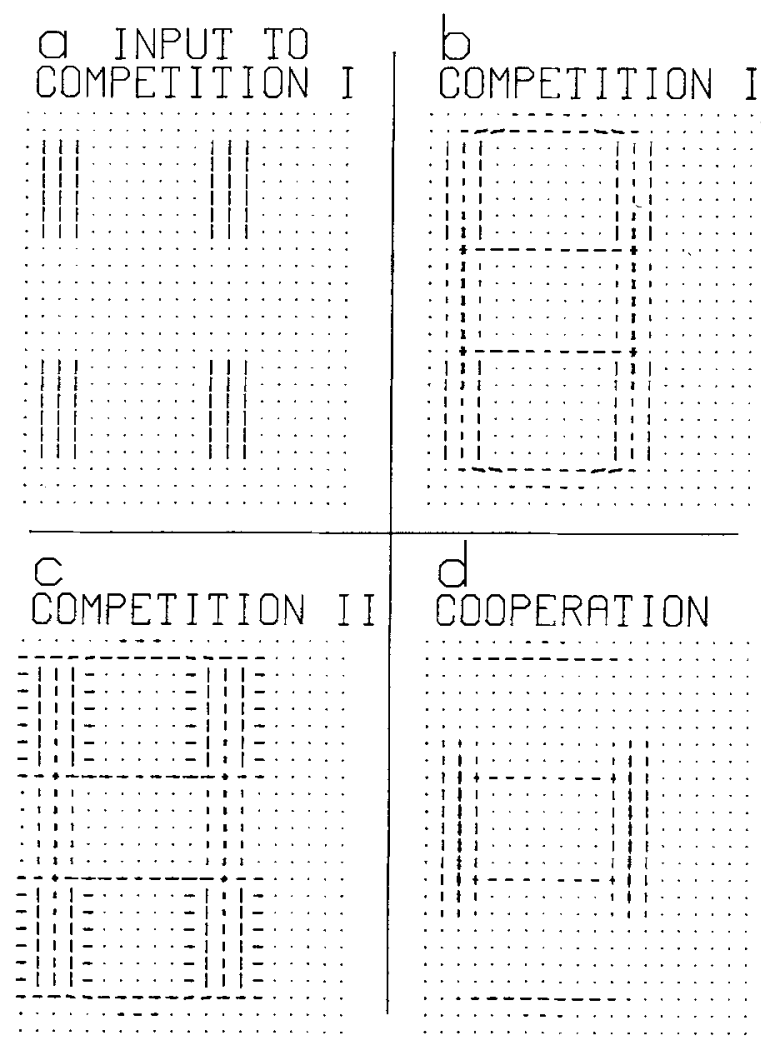

Figure 20. Coexistence of vertical and horizontal grouping: Here both horizontal and vertical groupings are completed at all Line ends.

agonal groupings emerge both on a microscopic scale and a macroscopic scale. Thus, diagonally oriented receptive fields are activated in the emergent boundaries, and these activations, as a whole, group into diagonal bands.

In Figure 23c, another shift of the inputs induces internal diagonal bands while enabling the exterior grouping into horizontal and diagonal boundaries to persist.

In Figure 24a, one of the vertical Lines is removed. The BC System now senses the remaining horizontal and diagonal symmetries (Figure 24c). In Figure 25a, the lower Line is moved further away from the upper pair of lines until the cooperation can no longer support the diagonal groupings. The diagonal groupings break apart, leaving the remaining horizontal groupings intact (Figure 25c).

\section{On-Line Statistical Decision Theory and Stochastic Relaxation}

These figures illustrate the fact that the BC System behaves like an on-line statistical decision theory in response to its input patterns. The BC System can sense only those groupings of perceptual elements which possess enough "statistical inertia" to drive its cooperative-competitive feedback exchanges toward a nonzero stable equilibrium configuration. The emergent patterns in Figures 18-25 are thus as important for what they do not show as they are for what they do show. All possible groupings of the 
oriented input elements could, in principle, have been generated, since all possible groupings of the cooperativecompetitive interaction were capable of receiving inputs.

In order to compare and contrast BC System properties with other approaches, one can interpret the distribution of oriented activities at each input position as being analogous to a local probability distribution, and the final BC System pattern as being the global decision that the system reaches and stores based upon all of its local data. The figures show that the BC System regards many of the possible groupings of these local data as spurious, and suppresses them as being functional noise. Some popular approaches to boundary segmentation and noise suppression do adopt a frankly probabilistic framework. For example, in a stochastic relaxation approach based upon statistical physics, Geman and Geman (1984) slowly decrease a formal temperature parameter that drives their system towards a minimal-energy configuration with boundary-enhancing properties. Zucker (1985) has also suggested a minimization algorithm to determine the best segmentation.

Such algorithms provide one way, indeed a classical way, to realize coherent properties within a many-body system. These algorithms define open-loop procedures in which external agents manipulate the parameters leading to coherence. In the BC System, by contrast, the only "ex-

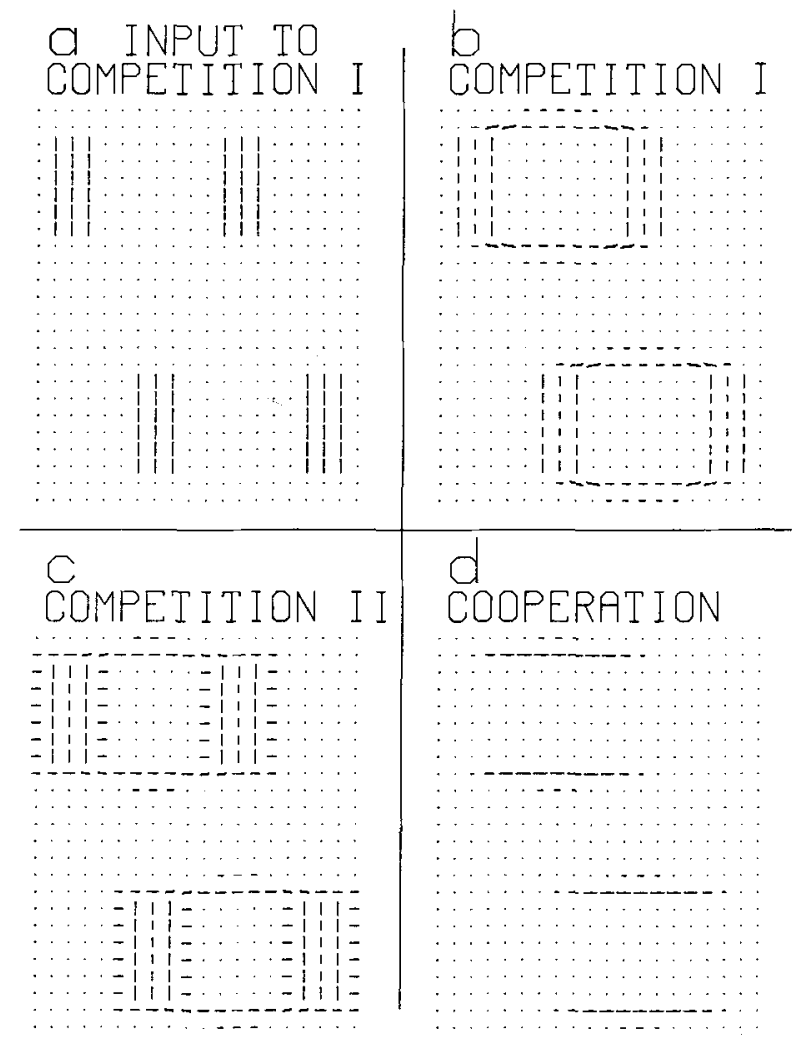

Figure 21. Horizontal grouping by end cuts: A horizontal shift of the lower two Lines in Figure 20 breaks the vertical groupings but preserves the horizontal groupings.

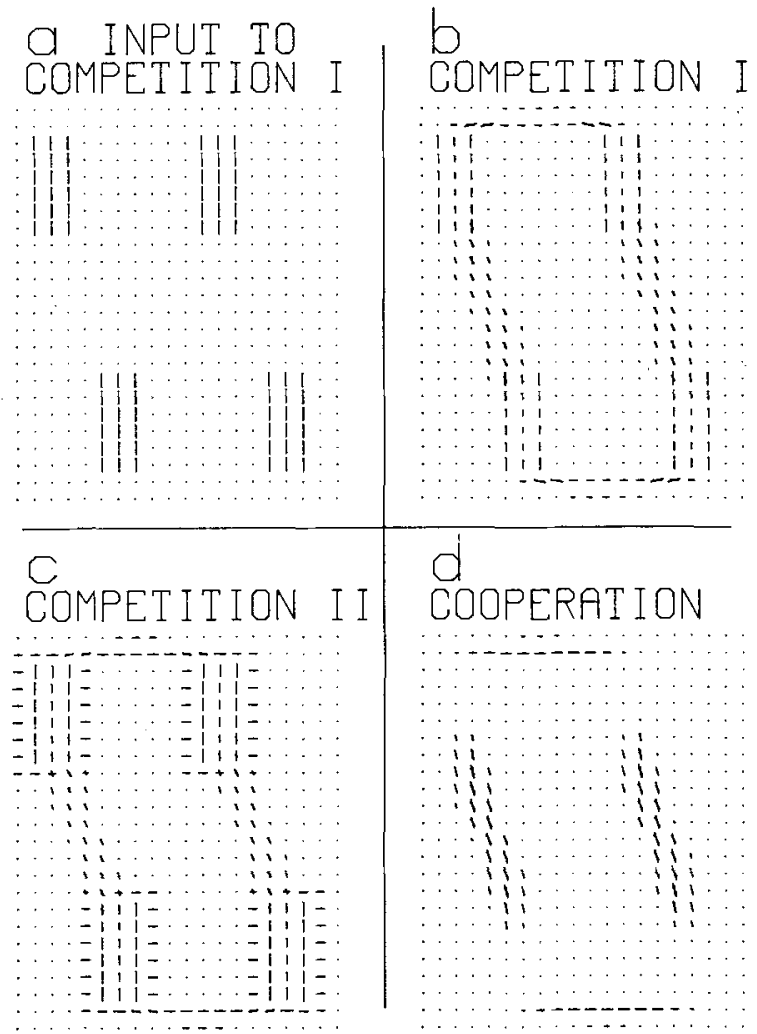

Figure 22. The emergence of diagonal groupings: The Boundary Contour System (BCS) is capable of generating groupings along orientations which have no activity in the oriented mask responses. Individual diagonally oriented cells are activated within the diagonally oriented groupings.

ternal parameters"' are the input patterns themselves. Each input pattern defines a different set of boundary conditions for the BC System, and this difference, in itself, generates different segmentations. The BC System does not need extra external parameters, because it contains a closed-loop process-the CC loop-which regulates its own convergence to a symmetric and coherent configuration via its real-time competitive-cooperative feedback exchanges.

The BC System differs in other major ways from alternative models. Geman and Geman (1984), for example; build into the probability distributions of their algorithm information about the images to be processed. The dynamics of the BC System clarify the relevance of probabilistic concepts to the segmentation process. In particular, the distributions of oriented activities at each input position (Figure 5) play the role of local probability distributions. On the other hand, within the BC System, these distributions emerge as part of a real-time reaction to input patterns, rather than according to predetermined constraints on probabilities. The BC System does not incorporate hypotheses about which images will be processed into its probability distributions. Such 
knowledge is not needed to achieve rapid preattentive segmentation.

The OR System does encode information about which images are familiar (Figure 1). Feedback interactions between the BC System and the OR System can rapidly supplement a preattentive segmentation using the templates read out from the OR System in response to BC System signals. Within our theory, however, these templates are not built into the OR System. Rather, we suggest how they are learned, in real time, as the OR System selforganizes its recognition code in response to the preattentively completed output patterns from the BC System (Carpenter \& Grossberg, 1985, in press; Grossberg, 1980, 1984b).

Thus, the present theory sharply distinguishes between the processes of preattentive segmentation and of learned object recognition. By explicating the intimate interaction between the BC System and the OR System, the present theory also clarifies why these distinct processes are often treated as a single process. In particular, the degree to which top-down learned templates can deform a preattentively completed BC System activity pattern will depend upon the particular images being processed and the past experiences of the OR System. Thus, by carefully selecting visual images, one can always argue that one, or the other process is rate-limiting. Furthermore, both

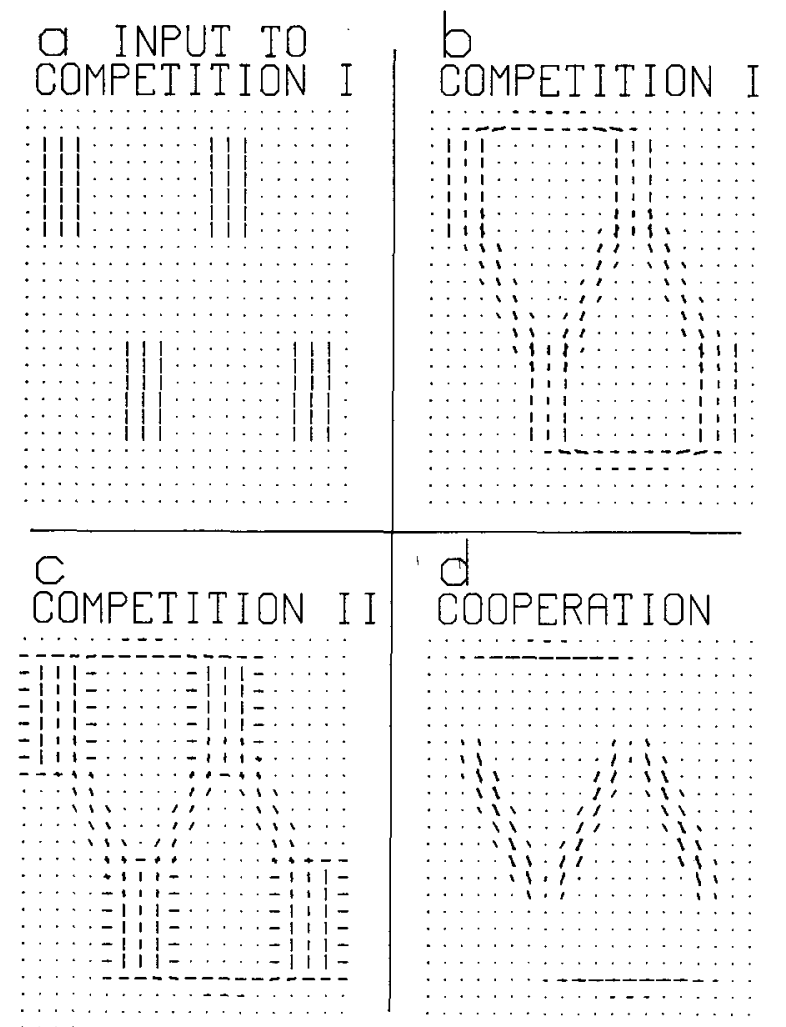

Figure 23. Multiple diagonal groupings: A new diagonal grouping emerges as a result of the shifting of input Lines. As in Figure 20, grouping in one orientation does not preclude grouping in an (almost) perpendicular orientation at the same Line end.

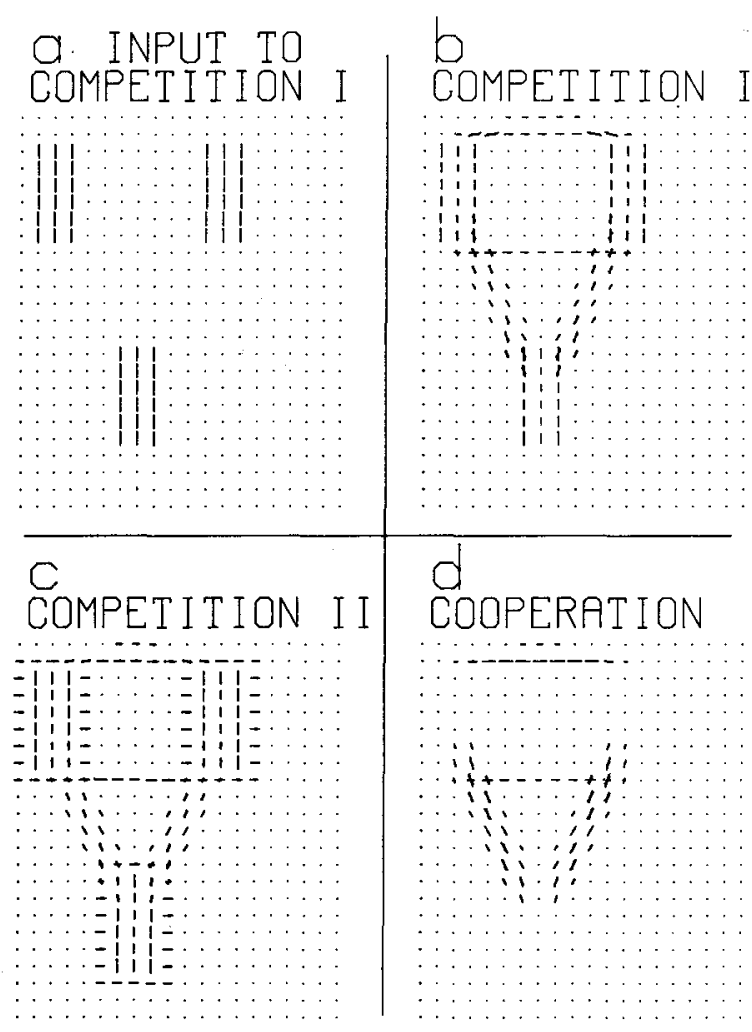

Figure 24. Global restructuring due to removal of local features: The inputs of this figure and those of Figure 23 are identical, except that the lower right $L$ ine has been removed. A comparison of Figure 24b and Figure 23b shows that, although gross aspects of the shared grouping are similar, removal of one Line can affect groupings among other Lines.

the preattentive BC System interactions and the top-down learned OR System interactions are processes of completion which enhance the coherence of BC System output patterns. They can thus easily be mistaken for one another.

\section{Correlations That Cannot Be Perceived: Simple Cells, Complex Cells, and Cooperation}

Glass and Switkes (1976) described a series of striking displays which they partially explained using the properties of cortical simple cells. Here we suggest a more complete explanation of their results using properties of the BC System. In their basic display (Figure 26), when "a random pattern of dots is superimposed on itself and rotated slightly ... a circular pattern is immediately perceived.... If the same pattern is superimposed on a negative of itself in which the background is a halftone gray and is rotated as before ..., it is impossible to perceive the circular Moiré. In this case spiral petal-like patterns can be seen"' (p. 67).

The circular pattern in Figure 26 is not "perceived" in an obvious sense. All that an observer can "see" are black dots on white paper. We suggest that the percept 


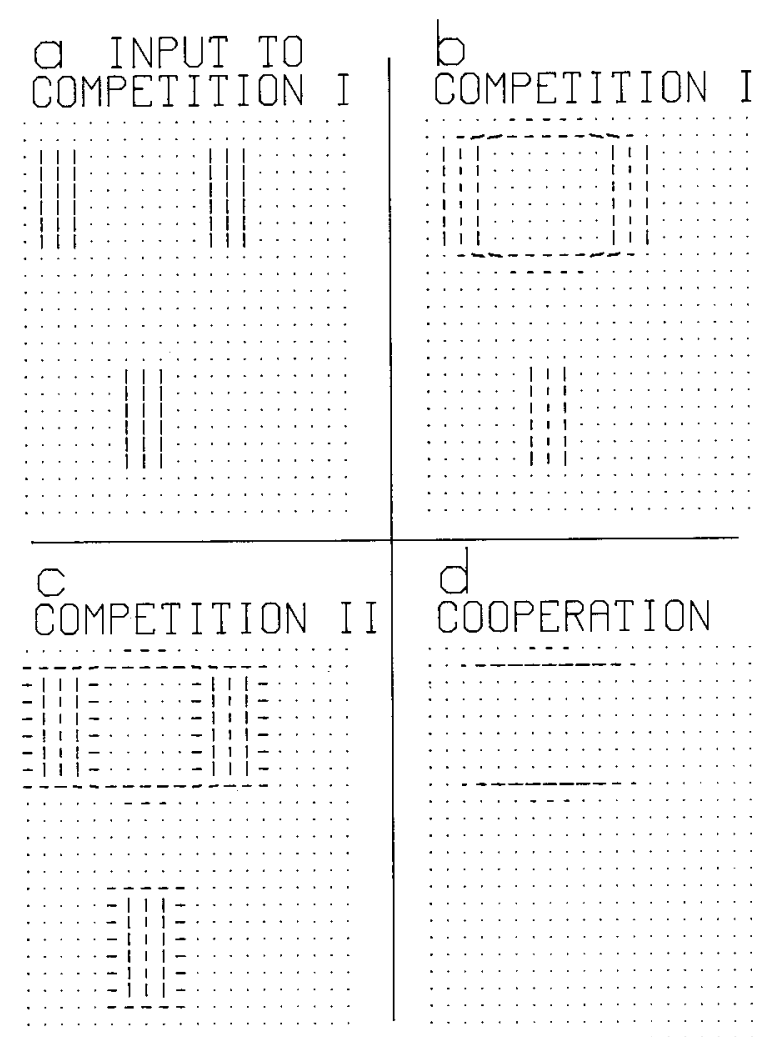

Figure 25. Distance-dependence of grouping: Relative to the inputs of Figure 24, the bottom Line has moved outside of the cooperative bandwidth that supported diagonal grouping. Although the diagonal grouping vanishes, the horizontal grouping at the bottom of the top Lines persists.

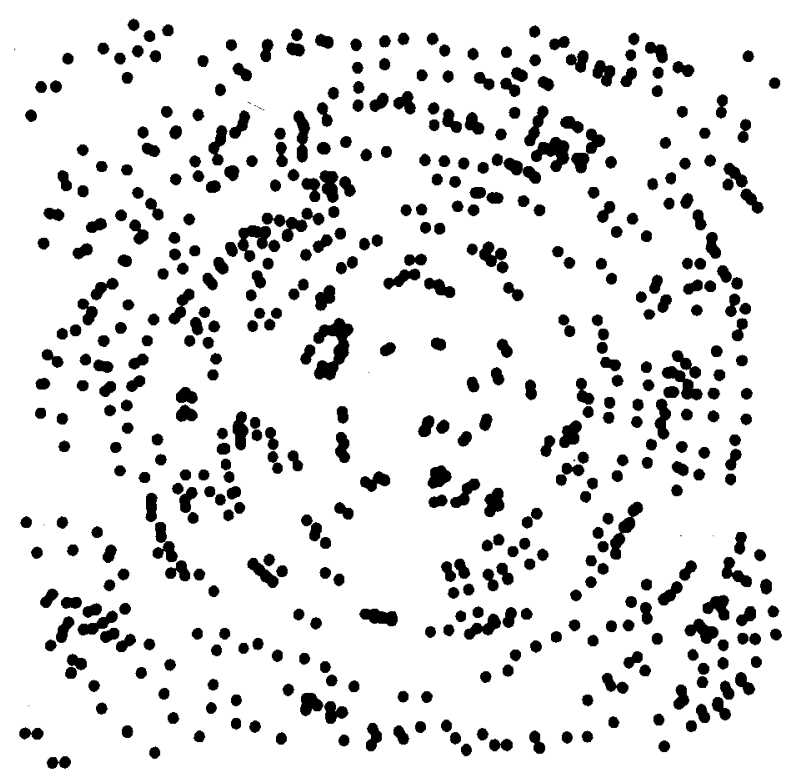

Figure 26. A Glass pattern: The emergent circular pattern is "recognized," although it is not "seen," as a pattern of differing contrasts. The text suggests how this can happen. (Reprinted, by permission, from Glass \& Switkes, 1975.) of circular structure is recognized by the OR System, whereas the FC System, wherein percepts of brightness and color are seen, generates the filled-in contrast differences that distinguish the black dots from the white background (Figure 1). A similar issue is raised by Figure 10, in which short vertical lines are seen even though emergent long vertical lines influence perceptual grouping. Thus, in the Glass and Switkes (1976) displays, no less than in the Beck et al. (1983) displays, one must sharply distinguish the recognition of perceptual groupings from the percepts that are seen. These recognition events always have properties of "coherence," whether or not they can support visible contrast differences. It then remains to explain why inverting the contrast of one of the images can alter what is recognized as well as what is seen.

We agree with part of the Glass and Switkes (1976) explanation. Consider a pair of black dots in Figure 26 that arises by rotating one image with respect to the other. Let the orientation of the pair with respect to the horizontal be $\theta^{\circ}$. Since the dots are close to one another, they can activate receptive fields that have an orientation approximately equal to $\theta^{\circ}$. This is due to the fact that an oriented receptive field is not an edge detector per se, but, rather, is sensitive to relative contrast differences across its medial axis. Only one of the two types of receptive fields at each position and orientation will be strongly activated, depending on the direction of contrast in the image. Each receptive field is sensitive to direction of contrast, even though pairs of these fields corresponding to like positions and orientations pool their activities at the next processing stage to generate an output that is insensitive to direction of contrast. We identify cells whose receptive fields are sensitive to direction of contrast with simple cells and the cells at the next stage which are insensitive to direction of contrast with complex cells of the striate cortex (DeValois, Albrecht, \& Thorell, 1982; Gouras \& Krüger, 1979; Heggelund, 1981; Hubel \& Wiesel, 1962, 1968; Schiller, Finlay, \& Volman, 1976; Tanaka, Lee, \& Creutzfeldt, 1983). Glass and Switkes (1976) did not proceed beyond this fact.

We suggest, in addition, that long-range cooperation within the BC System also plays a crucial role in grouping Glass images. To see how cooperation is engaged, consider two or more pairs of black dots that satisfy the following conditions: Each pair arises by rotating one image with respect to the other. The orientation of all pairs with respect to the horizontal is approximately $\theta^{\circ}$. All pairs are approximately collinear and do not lie too far apart. Such combinations of dots can more strongly activate the corresponding cooperative cells than can random combinations of dots. Each cooperative cell sends positive feedback to cells at the competitive stages with the same position and orientation. The competing cells that receive the largest cooperative signals gain an advantage over cells with different orientations. After competition among all possible cooperative groupings takes place, the favored groupings win and generate the large circular boundary contour structure that is recognized but not seen. Small circular boundaries are also generated around each 
dot and support the visible percept of dots on a white background within the FC System. Thus, the orientation $\theta^{\circ}$ of a pair of rotated black dots engages the BC System in two fundamentally different ways. First, it preferentially activates some oriented receptive fields above others. Second, it preferentially activates some cooperative cells above others due to combinations of inputs from preferentially activated receptive fields. As in the displays of Beck et al. (1983), the Glass images probe multiple levels of the BC System.

The other Glass images probe different levels of the BC System, notably the way in which simple cells activate complex cells which, in turn, activate the competitive layers. These images are constructed by reversing the contrast of one of the two images before they are superimposed. Then an observer sees black and white dots on a gray background. The recognition of circular macrostructure is, however, replaced by recognition of a more amorphous spiral petal-like pattern. Glass and Switkes (1976) noted that their "hypothesized neural mechanism does not appear to explain the observation of spiral-like patterns" (p. 71). To explain this recognition, we first note that the black dots on the gray background generate light-dark contrasts. Hence, the simple cells which responded to pairs of rotated black dots in Figure 26 are now stimulated by only one dot in each pair. Two or more randomly distributed black dots may be close enough to stimulate individual simple cells, but the orientations of the cells favored by stimulation by two or more random dots will be different from those of the cells stimulated by two or more rotated black dots in Figure 26. In addition, simple cells that are sensitive to the opposite direction of contrast can respond to the white dots on the gray background. These cells will be spatially rotated with respect to the cells that respond to the black dots. Moreover, since the black-to-gray contrast is greater than the white-to-gray contrast, the cells that respond to the black dots will fire more vigorously than the cells that respond to the white dots. Thus, although both classes of simple cells feed into the corresponding complex cells, the complex cells that respond to the black dots will be more vigorously activated than the complex cells that respond to the white dots. The cooperative stage will favor the most active combinations of complex cells whose orientations are approximately collinear and which are not too far apart. Due to the differences in spatial position and orientation of the most favored competitive cells, a boundary grouping different from that in Figure 26 is generated. A similar analysis can be given to the Glass and Switkes displays that use complementary colors.

In summary, the Glass and Switkes (1976) data emphasize three main points: Although simple cells sensitive to the same orientation and opposite direction of contrast feed into complex cells that are insensitive to direction of contrast, reversing the direction of contrast of some inputs can alter the positions and the orientations of the complex cells that are most vigorously activated. Although many possible groupings of cells can initially activate the cooperative stage, only the most favored groupings can survive the cooperative-competitive feedback exchange, as in Figures 18-25. Although all emergent boundary contours can influence the OR System, not all of these boundary countours can support visible filled-in contrast differences within the FC System. Prazdny (1984) has presented an extensive set of Glass-type displays, which have led him to conclude that "the mechanisms responsible for our perception of Glass patterns are also responsible for the detection of extended contours" (p. 476). Our theory provides a quantitative implementation of this assertion.

\section{Border Locking: The Café Wall Illusion}

A remarkable percept which is rendered plausible by BC System properties is the café wall illusion (Gregory \& Heard, 1979). This illusion is important because it clarifies the conditions under which the spatial alignment of collinear image contours with different contrasts is normally maintained. The illusion is illustrated in Figure 27.

The illusion occurs only if the luminance of the "mortar" within the horizontal strips lies between, or is not far outside, the luminances of the dark and light tiles, as in Figure 27. The illusion occurs, for example, in the limiting case of the Münsterberg figure, in which black and white tiles are separated by a black mortar. Gregory and Heard (1979) have also reported that the tile boundaries appear to "creep across the mortar during luminance changes"' (p. 368). Using a computer graphics system, we have generated a dynamic display in which the mortar luminance changes continuously through time. The perceived transitions from parallel tiles to wedge-shaped tiles and back are dramatic, if not stunning, using such a dynamic display.

Some of the BC System mechanisms that help to clarify this illusion can be inferred from Figure 28. This figure depicts a computer simulation of an orientation field that was generated in response to alternating black and white tiles surrounding a black strip of mortar. Figure 29 schematizes the main properties of Figure 28 . The hatched areas in Figure 29a depict the regions in which the greatest activations of oriented receptive fields occur. Due to the

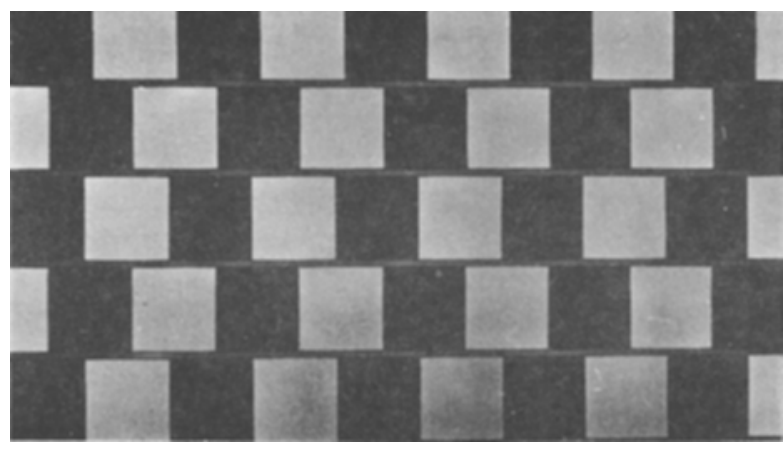

Figure 27. The café wall illusion: Although only horizontal and vertical luminance contours exist in this image, strong diagonal groupings are perceived. (Reprinted, by permission, from Gregory \& Heard, 1979). 
$\therefore: !: 3:$

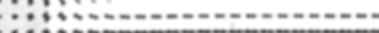
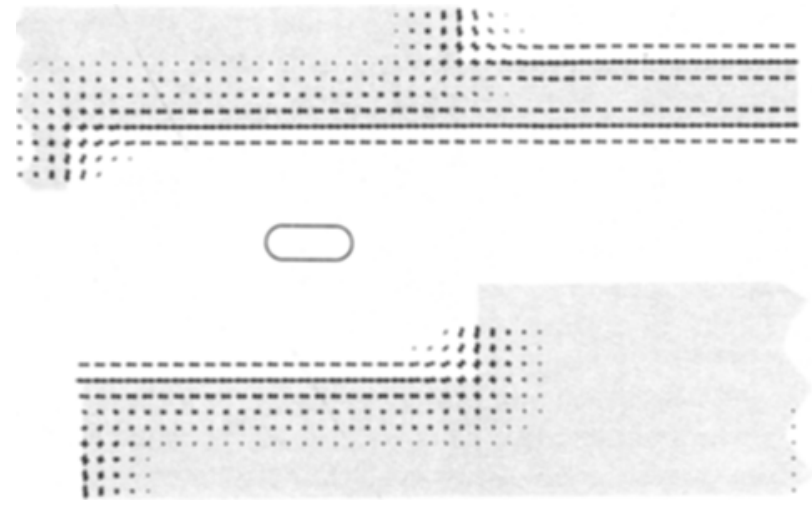

Figure 28. Simulation of the responses of a field of oriented masks to the luminance pattern near the mortar of the cafe wall illusion: The right of the bottom row joins to the left of the top row. The relative size of the masks used to generate the figure is indicated by the oblong shape in the center.

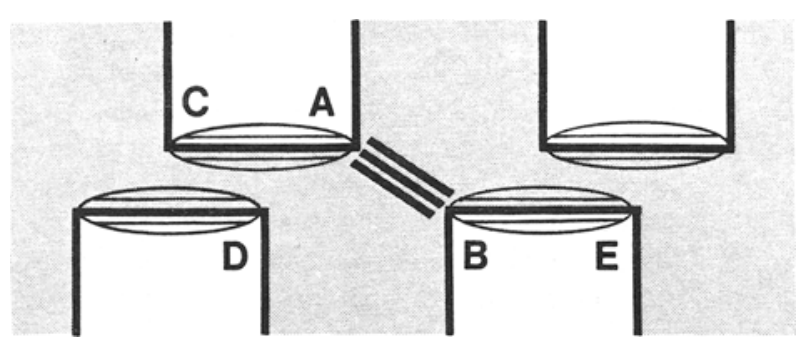

(a)

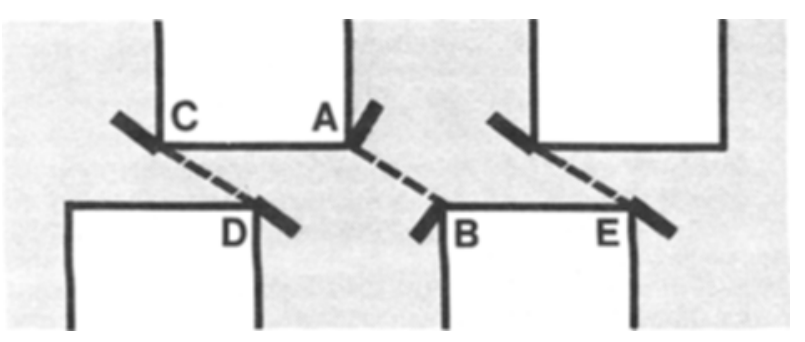

(b)

Figure 29. A schematic depiction of the simulation in Figure 28: (a) shows the region of strong horizontal activity and indicates a possible diagonal grouping between positions $A$ and $B$. (b) suggests that cooperation may occur in response to direct activations of oriented masks at positions $\mathrm{C}$ and $\mathrm{D}$, as well as in response to end cuts at positions $\mathrm{A}$ and $\mathrm{B}$. See text for additional discussion. approximately horizontal orientations of the activated receptive fields in Figure 29a, diagonal cooperative groupings between positions such as A and B can be initiated, as in Figures 22-24. Figure 28 thus indicates that a macroscopic spatial asymmetry in the activation of oriented receptive fields can contribute to the shifting of borders which leads to the wedge-shaped percepts.

Figure $29 \mathrm{~b}$ schematizes the fact that the microstructure of the orientation field is also skewed in Figure 28. Diagonal orientations tend to point into the black regions at the corners of the white tiles. Diagonal end cuts induced near positions A and B (Section 6) can thus cooperate between $A$ and $B$ in approximately the same direction as the macrostructure between $A$ and $C$ can cooperate with the macrostructure between $B$ and $E$ (Figure 29a). Diagonal activations near positions $C$ and $D$ can cooperate with each other in a direction almost parallel to the cooperation between A and B. These microscopic and macroscopic cooperative effects can help to make the boundaries at the top of the mortar seem to tilt diagonally downward.

Several finer points are clarified by the combination of these macroscale and microscale properties. By themselves, the microscale properties do not provide a sufficient explanation of why, for example, an end cut at position $\mathrm{D}$ cannot cooperate with direct diagonal activations at $\mathrm{A}$. The macroscale interactions tilt the balance in favor of cooperation between A and B. In the Münsterberg figure, the black mortar under a white tile may seem to glow, whereas the black mortar under a black tile does not. Using a dark-gray mortar, the gray mortar under a white tile may seem brighter, whereas the gray mortar under a black tile may better preserve its gray appearance. McCourt (1983) has also called attention to the relevance of brightness induction in explaining the café wall illusion. A partial explanation of these brightness percepts can be inferred from Figure 29. End cuts and diagonal groupings near position A may partially inhibit the parallel boundary between $A$ and $C$. Brightness can then flow from the white tile downward, as during neon color spreading (Figure 16). The more vigorous boundary activations above positions such as D and E (Figure 29a) may better contain local featural contrasts within a tighter web of boundary contours. This property also helps to explain the observation of Gregory and Heard (1979) that the white tiles seem to be pulled more into the black at such positions as $\mathrm{A}$ than at such positions as $\mathrm{C}$.

Our analysis of the café wall illusion, although not based on a complete computer simulation, suggests that the same three factors which play an important role in generating the Glass and Switkes (1976) data also play an important role in generating the Gregory and Heard (1979) data. In addition, perpendicular end cuts and multiple spatial scales seem to play a role in generating the Gregory and Heard (1979) data, with different combinations of scales acting between such positions as A-B than between such positions as C-D. This last property may explain why opposite sides $\mathrm{A}$ and $\mathrm{C}$ of an apparently wedge-shaped tile 
sometimes seem to lie at different depths from an observer (Grossberg, 1983b).

\section{Boundary Contour System Stages: \\ Predictions About Cortical Architectures}

This section outlines in greater detail the network interactions that we have used to characterize the BC System. Several of these interactions suggest anatomical and physiological predictions about the visual cortex. These predictions refine our earlier predictions that the data of von der Heydt et al. (1984) have since supported (Grossberg \& Mingolla, 1985a).

Figure 30 summarizes the proposed BC System interactions. The process whereby boundary contours are built up is initiated by the activation of oriented masks, or elongated receptive fields, at each position of perceptual space

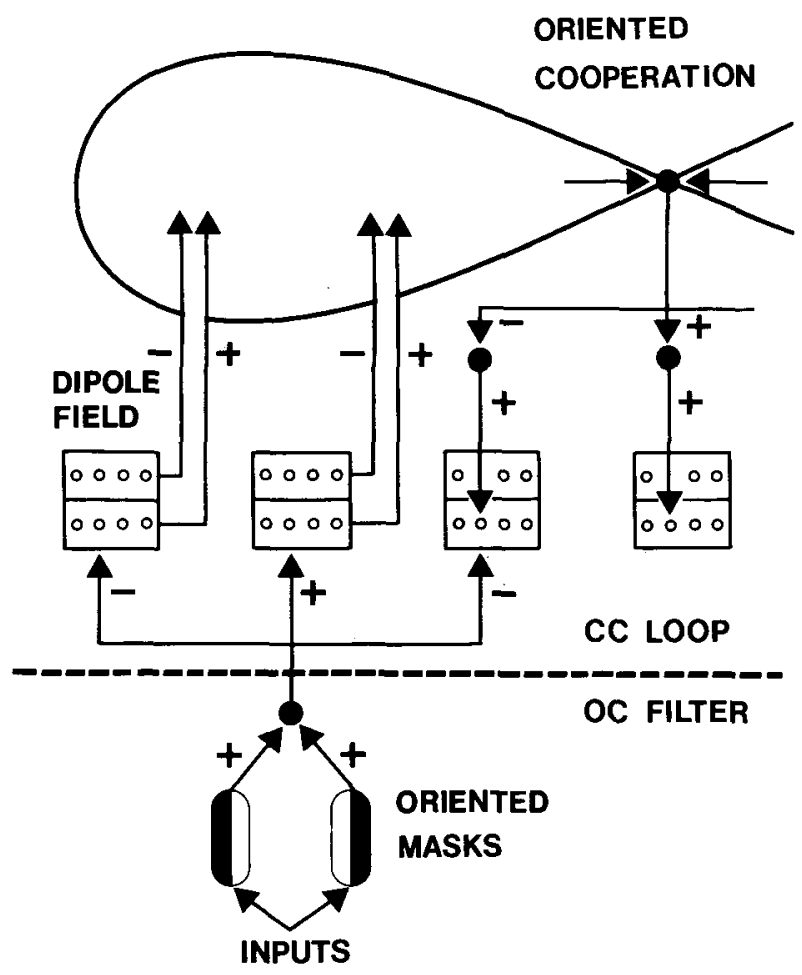

Figure 30. Circuit diagram of the BC System: Inputs activate oriented masks which cooperate at each position and orientation before feeding into an on-center-off-surround interaction. This interaction excites like-orientations at the same position and inhibits likeorientations at nearby positions. The affected cells are on-cells within a dipole field. On-cells at a fixed position compete among orientations. On-cells also inhibit off-cells which represent the same position and orientation. Off-cells at each position, in turn, compete among orientations. Both on-cells and off-cells are tonically active. Net excitation (inhibition) of an on-cell (off-cell) excites (inhibits) a cooperative receptive field corresponding to the same position and orientation. Sufficiently strong net positive activation of both receptive fields of a cooperative cell enables it to generate feedback via an on-center-off-surround interaction among like-oriented cells. Dipole on-cells which receive the most favorable combination of bottomup signals and top-down signals generate the emergent perceptual grouping.
(Hubel \& Wiesel, 1977). An oriented mask is a cell, or cell population, that is selectively responsive to oriented scenic-contrast differences. In particular, each mask is sensitive to scenic edges that activate a prescribed small region of the retina, and whose orientations lie within a prescribed band of orientations with respect to the retina. A family of such oriented masks lies at every network position, such that each mask is sensitive to a different band of edge orientations within its prescribed small region of the scene.

A. Position, orientation, amount of contrast, and direction of contrast. The first stage of oriented masks is sensitive to the position, orientation, amount of contrast, and direction of contrast at an edge of a visual scene. Thus, two subsets of masks exist corresponding to each position and orientation. One subset responds only to lightdark contrasts, and the other subset responds to dark-light contrasts. Such oriented masks do not, however, respond only to scenic edges. They can also respond to any image that generates a sufficiently large net contrast with the correct position, orientation, and direction of contrast within their receptive fields, as in Figures $14 \mathrm{~b}$ and 26. We identify these cells with the simple cells of striate cortex (DeValois et al., 1982; Hubel \& Wiesel, 1962, 1968; Schiller et al., 1976).

Pairs of oriented masks that are sensitive to similar positions and orientations but to opposite directions of contrast excite the next BC System stage. The output from this stage is thus sensitive to position, orientation, and amount of contrast, but is insensitive to direction of contrast. A vertical boundary contour can thus be activated by either a close-to-vertical light-dark edge or a close-tovertical dark-light edge at a fixed scenic position, as in Figure 3. The activities of these cells define the orientation field in Figure 5a. We identify the cells at this stage with the complex cells of striate cortex (DeValois et al., 1982; Gouras \& Krüger, 1979; Heggelund, 1981; Hubel \& Wiesel, 1962, 1968; Schiller et al., 1976; Tanaka et al., 1983). Spitzer and Hochstein (1985) have independently developed an essentially identical model of complex cell receptive fields to explain parametric properties of their cortical data.

B. On-center-off-surround interaction within each orientation. The outputs from these cells activate the first of two successive stages of short-range competition, which are denoted by Competition (I) and Competition (II) in Figures 18-25. At the first competitive stage, a mask of fixed orientation excites the like-oriented cells at its position and inhibits the like-oriented cells at nearby positions. Thus an on-center-off-surround interaction between like-oriented cells occurs around each perceptual location. This interaction predicts that a stage subsequent to striate complex cells organizes cells sensitive to like orientations at different positions so that they can engage in the required on-center-off-surround interaction.

C. Push-pull competition between orientations at each position. The inputs to the second competitive stage are the outputs from the first competitive stage. At the 
second competitive stage, competition occurs between different orientations at each position. Thus, a stage of competition between like orientations at different, but nearby, positions (Competition I) is followed by a stage of competition between different orientations at the same position (Competition II). This second competitive stage is tonically active. Thus, inhibition of a vertical orientation excites the horizontal orientation at the same position via disinhibition of its tonic activity.

The combined action of the two competitive stages generates the perpendicular end cuts in Figure $5 \mathrm{~b}$ that we have used to explain the percepts in Figures $7,8,12$, and 13. Conjoint inhibition of vertical and horizontal orientations by the first competitive stage leading to disinhibition of diagonal orientations at the second competitive stage (Figure 17) was also used to explain the diagonal groupings in Figure 15. A similar interaction was used to help explain the neon color-spreading phenomenon described in Figure 16 (Grossberg \& Mingolla, 1985a). Thus, the interactions of the first and second competitive stages help to explain a wide variety of seemingly unrelated perceptual groupings, color percepts, and illusory figures.

D. Dipole field: Spatial impenetrability. The process described in this section refines the BC System model that was used in Grossberg and Mingolla (1985a). This process incorporates a principle of cortical design that has been used to carry out related functional tasks in Grossberg $(1980,1983 a)$. The functional role played by this process in the BC System can be understood by considering Figure 18c.

At the second competitive stage of this figure, horizontal end cuts border the vertical responses to the inducing input Lines. What prevents the end cuts at both sides of each line from cooperating? If these end cuts could cooperate, then each Line could activate one of a cooperative cell's pair of receptive fields (Figure 9). As a result, horizontal boundary countours could be generated throughout the region between pairs of vertical Lines in Figure 18d, even though these Lines are spatially out-of-phase. The problem can thus be summarized as follows: Given the need for a long-range cooperative process to complete boundaries over retinal veins, the blind spot, and so forth, what prevents this cooperative process from leaping over intervening images and grouping together inappropriate combinations of inputs? In situations wherein no image-induced obstructions prevent such grouping, it can in fact occur, as in Figures 7 and 8 . If, however, cooperative grouping could penetrate all perceived objects, then many spurious groupings would occur across every Line. The perceptual space would be transparent with respect to the cooperative process.

To prevent this catastrophe, we propose a postulate of spatial impenetrability. This postulate suggests that mechanisms exist which prevent the cooperative process from grouping across all intervening percepts. Figure 18c discloses the primary computational properties that such a process must realize. It must not prevent like-oriented responses from cooperating in a spatially aligned position, because that is the primary functional role of cooperation. It need only prevent like-oriented responses (such as the horizontal end cuts in Figure 18a) from cooperating across a region of perpendicularly oriented responses (such as the vertical responses to the vertical Lines in Figure $18 \mathrm{c}$ ). We therefore hypothesize that the vertical responses to the Lines generate inhibitory inputs to horizontally oriented receptive fields of the cooperative process (Figure 31). The net input due to both horizontal end cuts and vertical lines at the horizontally oriented cooperative cells is thus very small or negative. As a result, neither receptive field of a horizontally oriented cooperative cell between the vertical Lines can be supraliminally excited. That is why the cooperative responses in Figure 18d ignore the horizontal end cuts.

It remains to say how both excitatory and inhibitory inputs are generated from the second competitive stage to the cooperative stage. We hypothesize that the second competitive stage is a dipole field (Grossberg, 1980, 1983a) and that inputs from the first competitive stage activate the on-cells of this dipole field. Suppose, for example, that an input excites vertically oriented on-cells, which inhibit horizontally oriented on-cells at the same position, as we have proposed in Section 20C. We assume, in addition, that inhibition of the horizontal on-cells excites the horizontal off-cells via disinhibition. The excited vertically oriented on-cells send excitatory inputs to the receptive fields of vertically oriented cooperative cells, whereas the excited horizontally oriented off-cells send

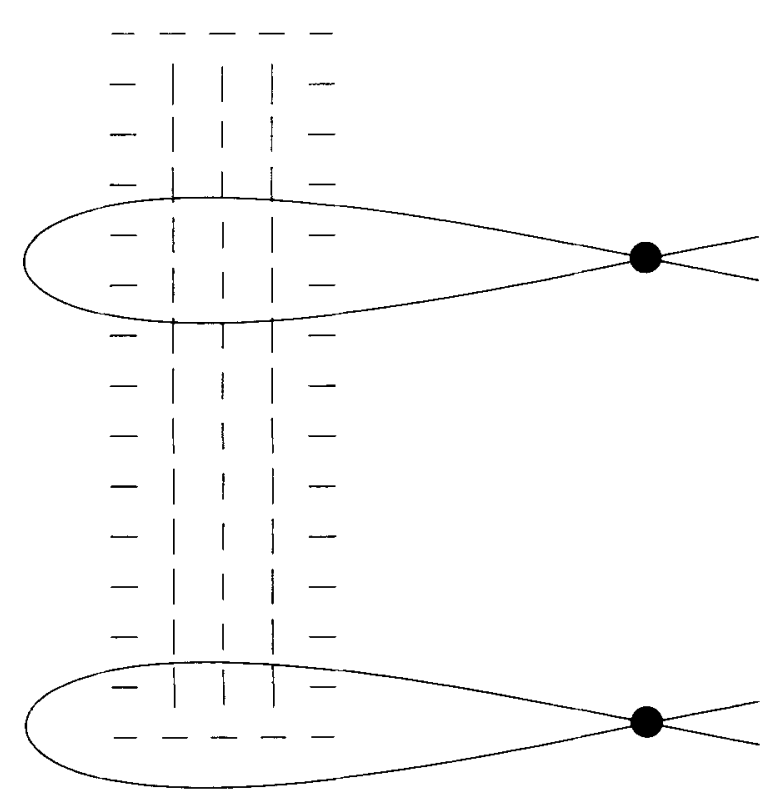

Figure 31. A mechanism to implement the postulate of spatial impenetability: The left receptive fields of two horizontally tuned cooperative cells are crossed by a thin vertical Line. Although horizontal end-cut signals can excite the upper receptive field, these are cancelled by the greater number of inhibitory inputs due to the vertical Line inputs. Within the lower receptive field, the excitatory inputs due to end cuts prevail. 
inhibitory inputs to the receptive fields of horizontally oriented cooperative cells (Figure 30 ).

Two new cortical predictions are implied by this dipolefield hypothesis: Both the on-cell subfield and the off-cell subfield of the dipole field are tonically active, thereby enabling their cells to be activated due to disinhibition. Excitation of on-cells generates excitatory inputs to likeoriented cooperative receptive fields, whereas excitation of off-cells generates inhibitory inputs to like-oriented cooperative receptive fields. The tonic activity of the oncell subfield helps to generate perpendicular end cuts, thereby preventing color flow from line ends. The tonic activity of the off-cell subfield helps to inhibit like-oriented cooperative cells, thereby augmenting spatial impenetrability.

E. Long-range oriented cooperation between likeoriented pairs of input groupings. The outputs from the dipole field input to a spatially long-range cooperative process. We call this process the boundary completion process. Outputs due to like-oriented dipole-field cells that are approximately aligned across perceptual space can cooperate via this process to synthesize an intervening boundary, as in Figures 18-25. A cooperative cell can be activated only if it receives a sufficiently positive net input at both of its orientationally tuned receptive fields (Figure 9).

Two types of parameters must be specified to characterize these receptive fields: macroscale parameters, which determine the gross shape of each receptive field,

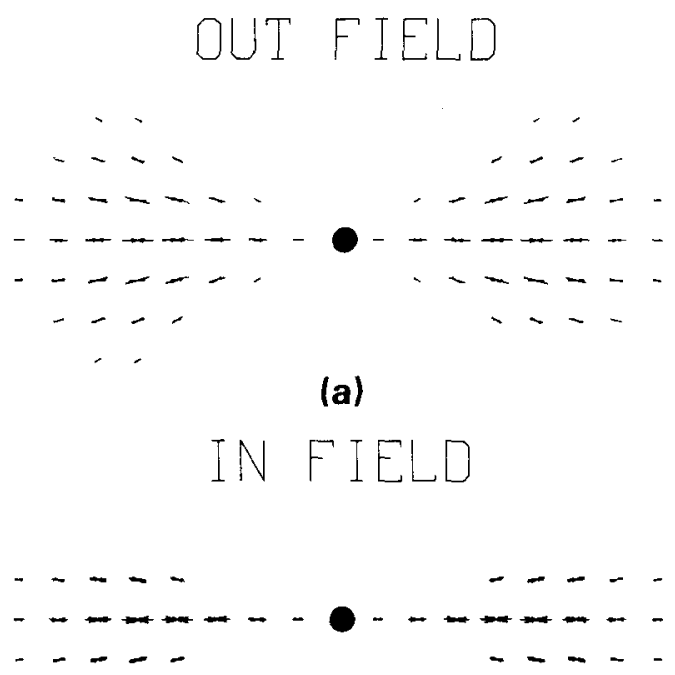

(b)

Figure 32. Cooperative in-field and out-field: Line lengths are proportional to the strengths of signals from a horizontally tuned competitive cell to cooperative cells of various orientations at nearby positions. Thus, in (a) strong signals are sent to horizontal cooperative cells 5 units to the left or the right of the competitive cell (center circle), but signal strength drops off with distance and change of orientation. (b) shows the dual perspective of weights assigned to incoming signals by the receptive field of a horizontal cooperative cell. (Note that only excitatory signal strengths are indicated in this figure.) The parameters used to generate these fields are identical to those used in Figures 18-25.

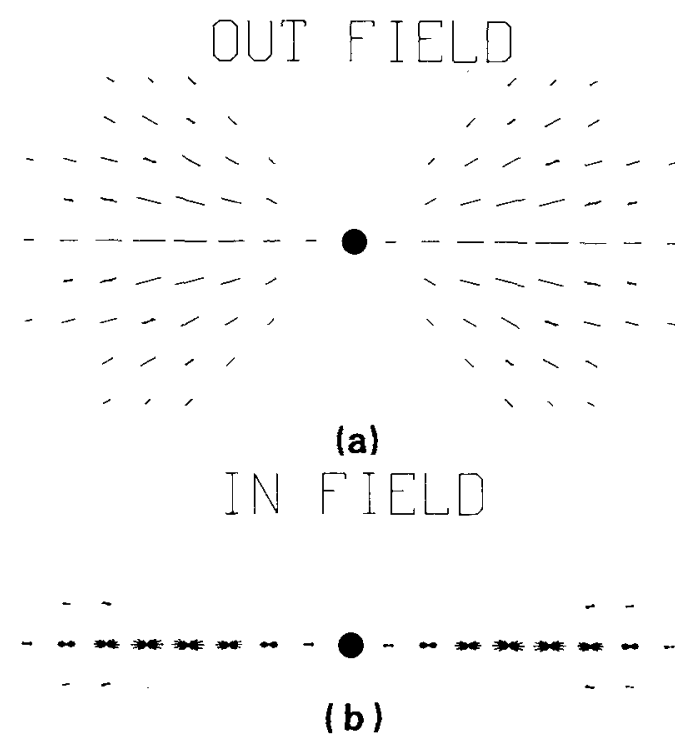

Figure 33. Extreme cooperative in-field and out-field: This figure employs more extreme parameter choices than were used in the simulations of Figure 18-25. Greater orientational uncertainty at one location of the in-field corresponds to greater positional uncertainty in the out-field, thereby illustrating the duality between in-field and out-field.

and microscale parameters, which determine how effectively a dipole-field input of prescribed orientation can excite or inhibit a cooperative receptive field. Figure 32 describes a computer simulation of the cooperative receptive field that we used to generate Figures 18-25. The cooperative out-field, or projection field, in Figure 32a describes the interaction strengths, or path weights, from a single horizontally oriented dipole-field on-cell to all cells within the cooperative stage. The length of each line is proportional to the size of the interaction strength to on-cells with the depicted positions and orientations. The cooperative in-field, or receptive field, in Figure 32b describes the path weights from all dipole-field on-cells with the depicted positions and preferred orientations to a single cooperative cell with a horizontally oriented receptive field. The length of each line is thus proportional to the sensitivity of the receptive field to inputs received from cells coding the depicted positions and orientations. The cell in Figure 32b is most sensitive to horizontally oriented inputs that fall along a horizontal axis passing through the cell. Close-to-horizontal orientations and close-to-horizontal positions can also help to excite the cell, but they are less effective. Figures 32a and $32 \mathrm{~b}$ describe the same information, but from different perspectives of a single dipole-field on-cell source (Figure 32a) and a single cooperative cell sink (Figure 32b).

Figure 33 depicts a cooperative out-field (Figure 33a) and in-field (Figure 33b) due to a different choice of numerical parameters. In Figure 33a, a single dipole-field on-cell can spray inputs over a spatially broad region, but the orientations that it can excite are narrowly tuned at each position. From the perspective of a cooperative cell's 
receptive fields, the out-field in Figure 33a generates an in-field that is spatially narrow, but the orientations that can excite it are broadly tuned. Figures 32 and 33 illustrate a duality between in-fields and out-fields that is made rigorous by the equations in the Appendix.

F. On-center-off-surround feedback within each orientation. This process refines the BC System that was described in Grossberg and Mingolla (1985a). In Section 8 , we suggested that excitatory feedback from the cooperative stage to the second competitive stage-more precisely to the on-cells of the dipole field-can help to eliminate fuzzy bands of boundaries by providing some orientations with a competitive advantage over other orientations. It is also necessary to provide some positions with a competitive advantage over other positions, so that only the favored orientations and positions will group to form a unique global boundary. Topographically organized excitatory feedback from a cooperative cell to a competitive cell is insufficient. Then the spatial fuzziness of the cooperative process (Figure 32) favors the same orientation at multiple noncollinear positions. Sharp orientational tuning but fuzzy spatial tuning of the resultant boundaries can then occur.

We suggest that the cooperative-to-competitive feedback process realizes a postulate of spatial sharpening in the following way. An active cooperative cell can excite likeoriented on-cells at the same position (Figure 30). An active cooperative cell can also inhibit like-oriented on-cells at nearby positions. Then both orientations and positions that are favored by cooperative groupings gain a competitive advantage within the on-cells of the dipole field.

Figures 18-25 show that the emergent groupings tend to be no thicker than the inducing input Lines due to this mechanism. Figure 30 shows that both the bottom-up inputs and the top-down inputs to the dipole field are organized as on-center-off-surround interactions among like orientations. The net top-down input is, however, always nonnegative due to the fact that excitatory interneurons are interpolated between the on-center-off-surround interaction and the dipole field. If this on-center-offsurround interaction were allowed to directly input to the dipole field, then a single line could generate a spatially expanding lattice of mutually perpendicular secondary, tertiary, and higher order end cuts via the cooperativecompetitive feedback loop. This completes our description of BC System interactions.

\section{Concluding Remarks: Universality of the Boundary Contour System}

The BC System and FC System interactions of our theory have suggested quantitative explanations and predictions for a large perceptual and neural data base, including data about perceptual grouping of textures and borders, illusory figures, monocular and binocular brightness percepts, monocular and binocular rivalry, the Land retinex demonstrations, neon color spreading and related filling-in phenomena, complementary color induction, fading of stabilized images, multiple scale interactions, shape-from-shading, metacontrast, hyperacuity, and var- ious other global interactions between depth, lightness, length, and form properties (Cohen \& Grossberg, 1984a; Grossberg, 1980, 1983a, 1984a, 1985; Grossberg \& Mingolla, 1985a).

This expanded explanatory and predictive range is due, we believe, to the introduction and quantitative analysis of several fundamental new principles and mechanisms to the perceptual literature, notably the principle of boundary-feature tradeoff and the mechanisms governing BC System and FC System interactions.

The present article has refined the mechanisms of the BC System by using this system to quantitatively simulate emergent perceptual grouping properties that are found in the data of such workers as Beck et al. (1983), Glass and Switkes (1976), and Gregory and Heard (1979). We have hereby been led to articulate and instantiate the postulates of spatial impenetrability and of spatial sharpening, and to thereby make some new predictions about prestriate cortical interactions. These results have also shown that several apparently different Gestalt rules can be analyzed using the context-sensitive reactions of a single BC System. Taken together, these results suggest that a universal set of rules for perceptual grouping of scenic edges, textures, and smoothly shaded regions is well on the way to being characterized.

\section{REFERENCES}

BECK, J. (1966a). Effect of orientation and of shape similarity on perceptual grouping. Perception \& Psychophysics, 1, 300-302.

BECK, J. (1966b). Perceptual grouping produced by changes in orientation and shape. Science, 154, 538-540.

BECK, J. (1972). Similarity grouping and peripheral discriminability under uncertainty. American Journal of Psychology, 85, 1-19.

BECK, J. (1982). Textural segmentation. In J. Beck (Ed.), Organization and representation in perception. Hillsdale, NJ: Erlbaum.

BECK, J. (1983). Textural segmentation, second-order statistics, and textural elements. Biological Cybernetics, 48, 125-130.

Beck, J., Prazdny, K., \& Rosenfeld, A. (1983). A theory of textural segmentation. In J. Beck, B. Hope, \& A. Rosenfeld (Eds.), Human and machine vision. New York: Academic Press.

CAELLI, T. (1982). On discriminating visual textures and images. Perception \& Psychophysics, 31, 149-159.

CAELLI, T. (1983). Energy processing and coding factors in texture discrimination and image processing. Perception \& Psychophysics, 34, 349-355.

Caelli, T., \& Dodwell, P. C. (1982). The discrimination of structure in vectorgraphs: Local and global effects. Perception \& Psychophysics, 32, 314-326.

Caelli, T., \&ulesz, B. (1979). Psychophysical evidence for global feature processing in visual texture discrimination. Joumal of the $O p$ tical Society of America, 69, 675-677.

Carpenter, G. A., \& Grossberg, S. (1981). Adaptation and transmitter gating in vertebrate photoreceptors. Journal of Theoretical Neurobiology, 1, 1-42.

Carpenter, G. A., \& Grossberg, S. (1983). Dynamic models of neural systems: Propagated signals, photoreceptor transduction, and circadian rhythms. In J. P. E. Hodgson (Ed.), Oscillations in mathematical biology. New York: Springer-Verlag.

Carpenter, G. A., \& Grossberg, S. (1985). Neural dynamics of category leaming and recognition: Attention, memory consolidation, and amnesia. In J. Davis, W. Newburgh, \& E. Wegman (Eds.), Brain structure, learning, and memory. Washington, DC: AAAS Symposium Series.

Carpenter, G. A., \& Grossberg, S. (in press). Neural dynamics of category learning and recognition: Structural invariants, evoked poten- 
tials, and reinforcement. In M. Commons, R. Herrnstein, \& S. Kosslyn (Eds.), Pattern recognition and concepts in animals, people, and machines. Hillsdale, NJ: Erlbaum.

Cohen, M. A., \& Grossberg, S. (1984a). Neural dynamics of brightness perception: Features, boundaries, diffusion, and resonance. Perception \& Psychophysics, 36, 428-456.

Cohen, M. A., \& Grossberg, S. (1984b). Some global properties of binocular resonances: Disparity matching, filling-in, and figure-ground synthesis. In P. Dodwell \& T. Caelli (Eds.), Figural synthesis. Hillsdale, NJ: Erlbaum.

Desimone, R., Schein, S. J., Moran, J., \& Ungerleider, L. G. (1985). Contour, color, and shape analysis beyond the striate cortex. Vision Research, 25, 441-452.

DEv, P. (1975). Perception of depth surfaces in random-dot stereograms: A neural model. International Journal of Man-Machine Studies, 7 , 511-528.

DeValois, R. L., Albrecht, D. G., \& Thorell, L. G. (1982). Spatial frequency selectivity of cells in macaque visual cortex. Vision Research, 22, 545-559.

DoDWELL, P. C. (1983). The Lie transformation group model of visual perception. Perception \& Psychophysics, 34, 1-16.

Ejima, Y., Redies, C., Takahashi, S., \& Akita, M. (1984). The neon color effect in the Ehrenstein pattern: Dependence on wavelength and illuminance. Vision Research, 24, 1719-1726.

Ellias, S., \& Grossberg, S. (1975). Pattern formation, contrast control, and oscillations in the short term memory of shunting on-center off-surround networks. Biological Cybernetics, 20, 69-98.

Geman, S., \& Geman, D. (1984). Stochastic relaxation, Gibbs distribution, and the Bayesian restoration of images. IEEE Patent Analysis \& Machine Intelligence, 6, 721-741.

Glass, L., \& SwITKES, E. (1976). Pattern recognition in humans: Correlations which cannot be perceived. Perception, 5, 67-72.

GoURAs, P., \&RÜGER, J. (1979). Responses of cells in foveal visual cortex of the monkey to pure color contrast. Journal of Neurophysiology, 42, 850-860.

GREGORY, R. L. (1966). Eye and brain. New York: McGraw-Hill.

GREGORY, R. L., \& HEARD, P. (1979). Border locking and the café wall illusion. Perception, 8, 365-380.

GrossberG, S. (1973). Contour enhancement, short-term memory, and constancies in reverberating neural networks. Studies in Applied Mathematics, 52, 217-257

Grossberg, S. (1980). How does a brain build a cognitive code? Psychological Review, 87, 1-51.

Grossberg, S. (1982). Studies of mind and brain: Neural principles of learning, perception, development, cognition, and motor control. Boston: Reidel Press.

GROSSBERG, S. (1983a). Neural substrates of binocular form perception: Filtering, matching, diffusion, and resonance. In E. Basar, $\mathbf{H}$. Flohr, H. Haken, \& A. J. Mandell (Eds.), Synergetics of the brain. New York: Springer

Grossberg, S. (1983b). The quantized geometry of visual space: The coherent computation of depth, form, and lightness. Behavioral \& Brain Sciences, 6, 625-692.

Grossberg, S. (1984a). Outline of a theory of brightness, color, and form perception. In E. Degreef \& J. van Buggenhaut (Eds.), Trends in mathematical psychology. Amsterdam: North-Holland.

GRossBerg, S. (1984b). Some psychophysiological and pharmacological correlates of a developmental, cognitive, and motivational theory. In R. Karrer, J. Cohen, \& P. Tueting (Eds.), Brain and information: Event related potentials. New York: New York Academy of Sciences.

GrossBerg, S. (1985). Cortical dynamics of depth, brightness, color, and form perception: A predictive synthesis. Manuscript submitted for publication.

Grossberg, S., \& LeVINE, D. (1975). Some developmental and attentional biases in the contrast enhancement and short term memory of recurrent neural networks. Journal of Theoretical Biology, $\mathbf{5 3}$, 341-380.

Grossberg, S., \& Mingolla, E. (1985a). Neural dynamics of form perception: Boundary completion, illusory figures, and neon color spreading. Psychological Review, 92, 173-211.
Grossberg, S., \& Mingolla, E. (1985b). Neural dynamics of surface perception: Boundary webs, illuminants, and shape-from-shading. Manuscript in preparation.

Heggelund, P. (1981). Receptive field organisation of complex cells in cat striate cortex. Experimental Brain Research, 42, 99-107.

Helmholtz, H. L. F. voN. (1962). Treatise on physiological optics (J. P. C. Southall, Trans.). New York: Dover. (Original work published 1890)

HofFMan, W. C. (1970). Higher visual perception as prolongation of the basic Lie transformation group. Mathematical Biosciences, 6 , 437-471.

Horn, B. K. P. (1977). Understanding image intensities. Artificial Intelligence, 8, 201-231.

HubeL, D. H., \& WIESEL, T. N. (1962). Receptive fields, binocular interaction and functional architecture in the cat's visual cortex. Journal of Physiology, 160, 106-154.

Hubel, D. H., \& WiEsel, T. N. (1968). Receptive fields and functional architecture of monkey striate cortex. Journal of Physiology, 195, 215-243.

HubEL, D. H., \& WIESEL, T. N. (1977). Functional architecture of macaque monkey visual cortex. Proceedings of the Royal Society of London $(B), 198,1-59$.

JuLESZ, B. (1960). Binocular depth perception of computer-generated patterns. Bell System Technical Journal, 39, 1125-1162.

Julesz, B. (1971). Foundations of cyclopean perception. Chicago: University of Chicago Press.

Kanisza, G. (1955). Margini quasi-percettivi in campi con stimolazione omogenea. Revista di psicologia, 49, 7-30.

KaPLAN, G. A. (1969). Kinetic disruption of optical texture: The perception of depth at an edge. Perception \& Psychophysics, 6, 193-198.

Kawabata, N. (1984). Perception at the blind spot and similarity grouping. Perception \& Psychophysics, 36, 151-158.

KenNeDY, J. M. (1979). Subjective contours, contrast, and assimilation. In C. F. Nodine \& D. F. Fisher (Eds.), Perception and pictorial representation (pp. 167-195). New York: Praeger

KrAUSKOPF, J. (1963). Effect of retinal image stabilization on the appearance of heterochromatic targets. Journal of the Optical Society of America, 53, 741-744.

LAND, E. H. (1977). The retinex theory of color vision. Scientific American, 237, 108-128.

Marr, D., \& Hildreth, E. (1980). Theory of edge detection. Proceedings of the Royal Society of London (B), 207, 187-217.

MarR, D., \& PoGgio, T. (1976). Cooperative computation of stereo disparity. Science, 194, 283-287.

McCourt, M. E. (1983). Brightness induction and the café wall illusion. Perception, 12, 131-142.

NeIsser, U. (1967). Cognitive psychology. New York: AppletonCentury-Crofts.

PRANDTL, A. (1927). Über gleichsinnige Induktion und die Lichtverteilung in gitterartigen Mustern. Zeitschrift für Sinnesphysiologie, 58, 263-307.

PrazDNy, K. (1983). Illusory contours are not caused by simultaneous brightness contrast. Perception \& Psychophysics, 34, 403-404.

Prazdny, K. (1984). On the perception of Glass patterns. Perception, 13, 469-478

PrazdNy, K. (1985). On the nature of inducing forms generating perception of illusory contours. Perception \& Psychophysics, 37, 237-242.

PREYER, W. (1897/1898). On certain optical phenomena: Letter to Professor E. C. Sanford. American Journal of Psychology, 9, 42-44.

RATLIFF, F. (1965). Mach bands: Quantitative studies on neural networks in the retina. New York: Holden-Day.

Redies, C., \& SpILlmanN, L. (1981). The neon color effect in the Ehrenstein illusion. Perception, 10, 667-681.

Redies, C., SpillmanN, L., \& Kunz, K. (1984). Colored neon flanks and line gap enhancement. Vision Research, 24, 1301-1309.

SCHATZ, B. R. (1977). The computation of immediate texture discrimination. MIT AI Memo 426.

Schiller, P. H., Finlay, B. L., \& Volman, S. F. (1976). Quantitative studies of single-cell properties in monkey striate cortex, I Spatiotemporal organization of receptive fields. Journal of Neurophysiology, 39, 1288-1319. 
Schwartz, E. L., Desimone, R., Albright, T., \& Gross, C. G (1983). Shape recognition and inferior temporal neurons. Proceedings of the National Academy of Sciences, 80, 5776-5778.

SHAPLEY, R., GoRDON, J. (1985). Nonlinearity in the perception of form. Perception \& Psychophysics, 37, 84-88.

SPERLING, G. (1970). Binocular vision: A physical and a neural theory. American Journal of Psychology, 83, 461-534.

SPILlMANN, L. (1985). Illusory brightness and contour perception: Current status and unresolved problems. Manuscript submitted for publication.

SPTitZer, H., \& HochSTEIN, S. (1985). A complex-cell receptive field model. Journal of Neurophysiology, 53, 1266-1286.

TANaka, M., LeE, B. B., \& CreutzFe LdT, O. D. (1983). Spectral tuning and contour representation in area 17 of the awake monkey. In J. D. Mollon \& L. T. Sharpe (Eds.), Colour vision. New York: Academic Press.

TODOROvić, D. (1983). Brightness perception and the Craik-O'BrienCornsweet effect. Unpublished MA thesis, University of Connecticut.

VaN TUxu, H. F. J. M. (1975). A new visual illusion: Neonlike color spreading and complementary color induction between subjective contours. Acta Psychologica, 39, 441-445.

vaN TUiJl, H. F. J. M., \& DE WeERT, C. M. M. (1979). Sensory conditions for the occurrence of the neon spreading illusion. Perception, $8,211-215$.

van TUIJL, H. F. J. M., \& Leeuwenberg, E. L. J. (1979). Neon color spreading and structural information measures. Perception \& Psychophysics, 25, 269-284.

von der Heydt, R., Peterhans, E., \& Baumgartner, G. (1984). Illusory contours and cortical neuron responses. Science, 224, 1260-1262.

WERTHEIMER, M. (1923). Untersuchungen zur Lehre von der Gestalt, II. Psychologische Forschung, 4, 301-350.

WOLFE, J. M. (1984). Global factors in the Hermann grid illusion. Perception, 13, 33-40.

Yarbus, A. L. (1967). Eye movements and vision. New York: Plenum Press.

ZEKI, S. (1983a). Colour coding in the cerebral cortex: The reaction of cells in monkey visual cortex to wavelengths and colours. Neuroscience, 9, 741-765.

ZEKI, S. (1983b) Colour coding in the cerebral cortex: The responses of wavelength-selective and colour coded cells in monkey visual cortex to changes in wavelength composition. Neuroscience, 9, 767-791.

ZUCKER, S. W. (1985). Early orientation selection: Tangent fields and the dimensionality of their support (Technical Report 85-13-R) Montreal: McGill University.

\section{APPENDIX}

\section{Boundary Contour System Equations}

The network we used to define the Boundary Contour System (BC System) is defined in stages below. This network further develops the BC System that was described in Grossberg and Mingolla (1985a).

\section{A. Oriented Masks}

To define a mask, or oriented receptive field, centered at position $(i, j)$ with orientation $k$, divide the elongated receptive field of the mask into a left-half $L_{i j k}$ and a right-half $R_{i j k}$. Let all the masks sample a field of preprocessed inputs. If $S_{p q}$ equals the preprocessed input to position $(p, q)$ of this field, then the output $\mathrm{J}_{\mathrm{ijk}}$ from the mask at position (i,j) with orientation $k$ is

$$
\mathbf{J}_{\mathrm{ijk}}=\frac{\left[\mathrm{U}_{\mathrm{ijk}}-\alpha \mathrm{V}_{\mathrm{ijk}}\right]^{+}+\left[\mathrm{V}_{\mathrm{ijk}}-\alpha \mathrm{U}_{\mathrm{ijk}}\right]^{+}}{1+\beta\left(\mathrm{U}_{\mathrm{ijk}}+\mathrm{V}_{\mathrm{ijk}}\right)},
$$

where

$$
\begin{aligned}
& \mathrm{U}_{\mathrm{ijk}}=\sum_{(\mathbf{p}, \mathrm{q}) \in \mathrm{L}_{\mathrm{j} j \mathrm{k}}} \mathrm{S}_{\mathbf{p q}}, \\
& \mathrm{V}_{\mathrm{ijk}}=\sum_{(\mathrm{p}, \mathrm{q}) \in \mathrm{R}_{\mathrm{ijk}}} \mathrm{S}_{\mathrm{pq}},
\end{aligned}
$$

and the notation $[p]^{+}=\max (p, 0)$. The sum of the two terms in the numerator of Equation $\mathrm{A} 1$ says that $\mathrm{J}_{\mathrm{ijk}}$ is sensitive to the orientation and amount of contrast, but not to the direction of contrast, received by $L_{i j k}$ and $R_{i j k}$. The denominator term in Equation Al enables $J_{i j k}$ to compute a ratio scale in the limit where $\beta\left(\mathrm{U}_{\mathrm{ijk}}+\mathrm{V}_{\mathrm{ijk}}\right)$ is much greater than 1 . In all of our simulations, we have chosen $\beta=0$.

\section{B. On-Center-Off-Surround Interaction \\ Within Each Orientation (Competition I)}

Inputs $\mathrm{J}_{\mathrm{ijk}}$ with a fixed orientation $\mathrm{k}$ activate potentials $\mathrm{w}_{\mathrm{ijk}}$ at the first competitive stage via on-center-off-surround interactions: each $J_{i j k}$ excites $w_{i j k}$ and inhibits $w_{p q k}$ if $|p-i|^{2}+|q-j|^{2}$ is sufficiently small. All the potentials $w_{i j k}$ are also excited by the same tonic input $\mathrm{I}$, which supports disinhibitory activations at the next competitive stage. Thus,

$$
\frac{d}{d t} w_{i j k}=-w_{i j k}+I+f\left(J_{i j k}\right)-w_{i j k} \sum_{(p, q)} f\left(J_{p q k}\right) A_{p q i j},
$$

where $A_{p q i j}$ is the inhibitory interaction strength between positions $(p, q)$ and $(i, j)$ and $f\left(J_{i j k}\right)$ is the input signal generated by $\mathrm{J}_{\mathrm{ijk}}$. In our runs, we chose

$$
f\left(J_{i j k}\right)=B J_{i j k} .
$$

Sections C and D together define the on-cell subfield of the dipole field described in Section 20.

\section{Push-Pull Opponent Processes \\ Between Orientation at Each Position}

Perpendicular potentials $w_{\mathrm{ijk}}$ and $w_{\mathrm{ijK}}$ elicit output signals that compete at their target potentials $\mathrm{x}_{\mathrm{ijk}}$ and $\mathrm{x}_{\mathrm{ijK}}$, respectively. For simplicity, we assume that these output signals equal the potentials $w_{i j k}$ and $w_{i j K}$, which are always nonnegative. We also assume that $\mathbf{x}_{\mathrm{ijk}}$ and $\mathbf{x}_{\mathrm{ijK}}$ respond quickly and linearly to these signals. Thus,

$$
\mathrm{x}_{\mathrm{ijk}}=\mathrm{w}_{\mathrm{ijk}}-\mathrm{w}_{\mathrm{ijk}}
$$

and

$$
x_{i j k}=w_{i j K}-w_{i j k} .
$$

\section{Normalization at Each Position}

We also assume that, as part of this push-pull opponent process, the outputs $y_{i j k}$ of the second competitive stage become normalized. Several ways exist for achieving this property (Grossberg, 1983b). We have used the following approach.

The potentials $\mathrm{x}_{\mathrm{ijk}}$ interact when they become positive. Thus, we let the output $\mathrm{O}_{\mathrm{ijk}}=\mathrm{O}\left(\mathrm{x}_{\mathrm{ijk}}\right)$ from $\mathrm{x}_{\mathrm{ijk}}$ equal

$$
\mathrm{O}_{\mathrm{ijk}}=\mathrm{C}\left[\mathrm{w}_{\mathrm{ijk}}-\mathrm{w}_{\mathrm{ijk}}\right]^{+} \text {, }
$$


where $C$ is a positive constant and $[p]^{*}=\max (p, 0)$. All these outputs at each position interact via a shunting on-center-offsurround network whose potentials $y_{i j k}$ satisfy

$$
\frac{d}{d t} y_{i j k}=-D y_{i j k}+\left(E-y_{i j k}\right) O_{i j k}-y_{i j k} \sum_{m \neq k} O_{i j m} .
$$

Each potential $y_{i j k}$ equilibrates rapidly to its input. Setting

$$
\frac{d}{d t} y_{i j k}=0
$$

in Equation $\mathrm{A} 9$ implies that

$$
y_{i j k}=\frac{E O_{i j k}}{D+O_{i j}},
$$

where

$$
\mathrm{O}_{\mathrm{ij}}=\sum_{\mathrm{m}=1}^{\mathbf{n}} \mathrm{O}_{\mathrm{ijm}}
$$

Thus, if $D$ is small compared with $O_{i j}$, then $\sum_{m=1}^{n} y_{i j m} \cong E$.

\section{E. Opponent Inputs to the Cooperative Stage}

The next process refines the BCS model used in Grossberg and Mingolla (1985a). It helps to realize the postulate of spatial impenetrability that was described in Section 20 . The $w_{i j k}, x_{i j k}$, and $y_{i j k}$ potentials are all assumed to be part of the on-cell subfield of a dipole field. If $y_{i j k}$ is excited, an excitatory signal $f\left(y_{i j k}\right)$ is generated at the cooperative stage. When potential $y_{i j k}$ is excited, the potential $\mathrm{y}_{\mathrm{ijK}}$ corresponding to the perpendicular orientation is inhibited. Both of these potentials form part of the oncell subfield of a dipole field. Inhibition of an on-cell potential $y_{i j k}$ disinhibits the corresponding off-cell potential $\bar{y}_{i j K}$, which sends an inhibitory signal $-f\left(\bar{y}_{i j k}\right)$ to the cooperative level. The signals $f\left(y_{i j k}\right)$ and $-f\left(\bar{y}_{i j k}\right)$ thus occur together. In order to instantiate these properties, we made the simplest hypothesis, namely that

$$
\bar{y}_{\mathrm{ijk}}=\mathrm{y}_{\mathrm{ijk}} .
$$

\section{F. Oriented Cooperation: Statistical Gates}

The cooperative potential $\mathrm{z}_{\mathrm{ijk}}$ can be supraliminally activated only if both of its cooperative input branches receive enough net positive excitation from similarly aligned competitive potentials (Figure 9). Thus,

$$
\begin{aligned}
\frac{d}{d t} z_{i j k}= & -z_{i j k}+g\left(\sum_{(\mathbf{p}, q, r)}\left[f\left(y_{p q r}\right)-f\left(\bar{y}_{p q r}\right)\right] F_{p q i j}^{(r, k)}\right) \\
& +g\left(\sum_{(p, q, r)}\left[f\left(y_{p q r}\right)-f\left(\bar{y}_{p q r}\right)\right] G_{p q i j}^{(r, k)}\right) .
\end{aligned}
$$

In Equation $\mathrm{A} 13, \mathrm{~g}(\mathrm{~s})$ is a signal function that becomes positive only when $s$ is positive, and has a finite maximum value. A slower-than-linear function,

$$
g(s)=\frac{H[s]^{+}}{K+[s]^{+}},
$$

was used in our simulations. A sum of two sufficiently positive $\mathrm{g}(\mathrm{s})$ terms in Equation A13 is needed to activate $\mathrm{z}_{\mathrm{ijk}}$ above the firing threshold of its output signal $h\left(\mathrm{z}_{\mathrm{ijk}}\right)$. A threshold-linear signal function,

$$
\mathrm{h}(\mathrm{z})=\mathrm{L}[\mathrm{z}-\mathrm{M}]^{+},
$$

was used. Each sum, such as

$$
\sum_{(p, q, r)} f\left(y_{p q r}\right) F_{p q i j}^{(r, k)}
$$

and

$$
\sum_{(\mathbf{p}, q, \mathrm{r})} f\left(y_{\mathbf{p q r}}\right) \mathrm{G}_{\mathbf{p q i j}}^{(\mathbf{r}, \mathbf{k})}
$$

is a spatial cross-correlation that adds up inputs from a strip with orientation (approximately equal to) $\mathbf{k}$, which lies to one side or the other of position $(i, j)$, as in Figures 32 and 33 . The orientations $r$ that contribute to the spatial kernels $F_{p q i j}^{(r, k)}$ and $G_{p q i j}^{(r, k)}$ also approximately equal $k$. The kernels $F_{p q i j}^{(r, k)}$ and $G_{p q i j}^{(r, k)}$ are defined by

$$
\begin{aligned}
F_{p q i j}^{(I, k)}= & {\left[\exp \left[-2\left(N_{p q i j} P^{-1}-1\right)^{2}\right]\right.} \\
& \left.\times\left[\left|\cos \left(Q_{p q i j}-r\right)\right|\right]^{R^{R}}\left[\cos \left(Q_{p q i j}-k\right)\right]^{T}\right]^{+}
\end{aligned}
$$

and

$$
\begin{aligned}
\mathrm{G}_{\mathrm{pqij}}^{(\mathrm{r}, \mathrm{k})}= & {\left[-\exp \left[-2\left(\mathrm{~N}_{\mathrm{pqij}} \mathrm{P}^{-1}-1\right)^{2}\right]\right.} \\
& \left.\times\left[\left|\cos \left(\mathrm{Q}_{\mathrm{pqij}}-\mathrm{r}\right)\right|\right]^{\mathrm{R}}\left[\cos \left(\mathrm{Q}_{\mathrm{pqij}}-\mathrm{k}\right)\right]^{\mathrm{T}}\right]^{+},
\end{aligned}
$$

where

$$
\begin{gathered}
N_{p q i j}=\sqrt{(p-i)^{2}+(q-j)^{2}}, \\
Q_{p q i i}=\arctan \left(\frac{q-j}{p-i}\right),
\end{gathered}
$$

and $P, R$, and $T$ are positive constants. In particular, $R$ and $T$ are odd integers. Kernels $F$ and $\mathbf{G}$ differ only by a minus sign under the $[\cdots]^{+}$sign. This minus sign determines the polarity of the kernel, namely, whether it collects inputs for $\mathrm{z}_{\mathrm{ijk}}$ from one side or the other of position $(i, j)$. Term

$$
\exp \left[-2\left(\frac{N_{p q i j}}{P}-1\right)^{2}\right]
$$

determines the optimal distance $P$ from $(i, j)$ at which each kernel collects its inputs. The kernel decays in a Gaussian fashion as a function of $N_{p q i j} / P$, where $N_{p q i j}$ in Equation A20 is the distance between $(p, q)$ and $(i, j)$. The cosine terms in Equations A18 and $A 19$ determine the orientational tuning of the kernels. By Equation $A 21, Q_{p q i j}$ is the direction of position $(p, q)$ with respect to the position of the cooperative cell $(i, j)$ in Equation A13. Term $\left|\cos \left(Q_{p q i j}-r\right)\right|$ in Equations $A 18$ and $A 19$ computes how parallel $Q_{p q i j}$ is to the receptive field orientation $r$ at position $(p, q)$. By Equation $A 21$, term $\left|\cos \left(Q_{p q i j}-r\right)\right|$ is maximal when the orientation $r$ equals the orientation of $(p, q)$ with respect to $(\mathrm{i}, \mathrm{j})$. The absolute value sign around this term prevents it from becoming negative. Term $\cos \left(Q_{p q i j}-k\right)$ in Equations $A 18$ and A 19 computes how parallel $Q_{p q i j}$ is to the orientation $k$ of the receptive field of the cooperative cell $(i, j)$ in Equation A13. By 
Equation $\mathrm{A} 21$, term $\cos \left(\mathrm{Q}_{\mathrm{pqij}}-\mathrm{k}\right)$ is maximal when the orientation $k$ equals the orientation of $(p, q)$ with respect to $(i, j)$. Positions $(p, q)$ such that $\cos \left(Q_{p q i j}-k\right)<0$ do not input to $z_{i j k}$ via kernel $\mathrm{F}$ because the $[\cdots]^{+}$of a negative number equals zero. On the other hand, such positions $(p, q)$ may input to $z_{i j k}$ via kernel $G$ due to the extra minus sign in the definition of kernel $G$. The extra minus sign in Equation A19 flips the preferred axis of orientation of kernel $G_{p q i j}^{(r, k)}$ with respect to the kernel $F_{p q i j}^{(r, k)}$ in order to define the two input-collecting branches of each cooperative cell, as in Figures 9 and 30. The product terms $\left[\left|\cos \left(Q_{p q i i}-r\right)\right|\right]^{R}\left[\cos \left(Q_{p q i j}-k\right)\right]^{T}$ in Equations A18 and A19 thus determine larger path weights from dipole field on-cells whose positions and orientations are nearly parallel to the preferred orientation $k$ of the cooperative cell $(i, j)$, and larger path weights from dipole-field off-cells whose positions and orientations are nearly perpendicular to the preferred orientation $k$ of the cooperative cell $(i, j)$. The powers $R$ and $T$ determine the sharpness of orientational tuning: Higher powers enforce sharper tuning.

\section{G. On-Center-Off-Surround Feedback Within Each Orientation}

The next process refines the BC System model used in Grossberg and Mingolla (1985). It helps to realize the postulate of spatial sharpening that was described in Section 20. We assume that each $\mathrm{z}_{\mathbf{i j k}}$ activates a shunting on-center-off-surround interaction within each orientation $k$. The target potentials $v_{i j k}$ therefore obey an equation of the form

$$
\frac{d}{d t} v_{i j k}=-v_{i j k}+h\left(z_{i j k}\right)-v_{i j k} \sum_{(p, q)} h\left(z_{p q k}\right) W_{p q i j} .
$$

The bottom-up transformation $J_{i j k} \rightarrow w_{i j k}$ in Equation A4 is thus similar to the top-down transformation $z_{i j k} \rightarrow v_{i j k}$ in Equation A22. Functionally, the $z_{i j k} \rightarrow v_{i j k}$ transformation enables the most favored cooperations to enhance their preferred positions and orientation as they suppress nearby positions with the same orientation. The signals $v_{i j k}$ take effect by inputting to the $\mathbf{w}_{\mathrm{ijk}}$ opponent process. Equation A4 is thus changed to

$$
\frac{d}{d t} w_{i j k}=-w_{i j k}+I+f\left(J_{i j k}\right)+v_{i j k}-w_{i j k} \sum_{(p, q)} f\left(J_{p q k}\right) A_{p q i j} .
$$

At equilibrium, the computational logic of the BC System is determined, up to parameter choices, by the equations

$$
\begin{aligned}
& J_{i j k}=\frac{\left[U_{i j k}-\alpha V_{i j k}\right]^{+}+\left[V_{i j k}-\alpha U_{i j k}\right]^{+}}{1+\beta\left(U_{i j k}+V_{i j k}\right)}, \\
& w_{i j k}=\frac{I+B J_{i j k}+v_{i j k}}{1+B \Sigma_{(p, q)} J_{p q k} A_{p q i j}}, \\
& \mathrm{O}_{\mathrm{ijk}}=\mathrm{C}\left[\mathrm{w}_{\mathrm{ijk}}-\mathrm{w}_{\mathrm{ijk}}\right]^{+} \text {, } \\
& y_{i j k}=\frac{E O_{i j k}}{D+O_{i j}} \text {, } \\
& z_{i j k}=g\left(\sum_{(p, q, r)}\left[f\left(y_{p q r}\right)-f\left(y_{p q R}\right)\right] F_{p q i j}^{(r, k)}\right) \\
& +g\left(\sum_{(p, q, r)}\left[f\left(y_{p q r}\right)-f\left(y_{p q R}\right)\right] G_{p q i j}^{(r, k)}\right),
\end{aligned}
$$

and

$$
v_{i j k}=\frac{h\left(z_{i j k}\right)}{1+\Sigma_{(p, q)} h\left(z_{p q k}\right) W_{p q i j}} .
$$

Wherever possible, simple spatial kernels were used. For example, the kernels $W_{\text {pqij }}$ in Equation A22 and $A_{\text {pqij }}$ in Equation A23 were both chosen to be constant within a circular receptive field:

$$
A_{p q i j}= \begin{cases}A & \text { if }(p-i)^{2}+(q-j)^{2} \leq A_{0} \\ 0 & \text { otherwise }\end{cases}
$$

and

$$
W_{p q i j}= \begin{cases}W & \text { if }(p-i)^{2}+(q-j)^{2} \leq W_{0} \\ 0 & \text { otherwise. }\end{cases}
$$

(Manuscript received April 4, 1985; revision accepted for publication August 9, 1985.) 